\title{
CFD analysis of cross-ventilation flow in a group of generic buildings: Comparison between steady RANS, LES and wind tunnel experiments
}

\begin{abstract}
Computational fluid dynamics (CFD) results generated by the steady Reynolds-averaged Navier-Stokes equations (SRANS) model and large eddy simulation (LES) are compared with wind tunnel experiments for investigating a cross-ventilation flow in a group of generic buildings. The mean flow structure and turbulence statistics are compared for SRANS based on different two-equation turbulence models with LES based on the Smagorinsky subgrid-scale turbulence model. The LES results show very close agreement with the experimental results in the prediction of the time-averaged velocity, wind surface pressure around and inside the building, and crossing flow through the openings. In contrast, SRANS fails to predict the most important features of cross-ventilation. LES reproduced well the anisotropic turbulence property around and inside the cross-ventilated building, which is closely related to the transient momentum transfer caused in street canyon flows and has a significant influence on the mean flow structure. In contrast, SRANS could not inherently reproduce such transient fluctuations and anisotropic turbulence property, and it results in low accurate predictions for the time-average velocity components, wind surface pressure distribution and crossing airflow rate up to $100 \%$ error.
\end{abstract}

Keywords: CFD, Steady RANS, LES, wind tunnel experiment, cross-ventilation, sheltered building

\section{Introduction}

Wind induced cross-ventilation has been utilized in traditional and modern buildings for air-quality improvement (Lien and Ahmed 2011; Heracleous and Michael 2019; Aflaki et al. 2019; Aydin and Mirzaei 2016) and building energy reduction (Mochida et al. 2006; Mohammadreza Shirzadi, Naghashzadegan, and Mirzaei 2019; Zhang, Mirzaei, and Jones 2018; Ohba and Lun 2010; Lo and Novoselac 2013; Li et al. 2014). Different methods are used for analyzing cross-ventilation, including simplified correlations based on the orifice flow theory (Kobayashi et al. 2009; Takashi Kurabuchi et al. 2005; Karava, Stathopoulos, and Athienitis 2004; Martins and da Graça 2016), field measurement (H. Gough et al. 2018; Park 2013; Mochida et al. 2005), wind tunnel measurement (Tominaga and Blocken 2016; Mohammadreza Shirzadi, Tominaga, and Mirzaei 2019c; Katayama, Tsutsumi, and Ishii 1992; C.-R. Chu and Chiang 2014; C. R. Chu, Chiu, and Wang 2010; Tominaga and Blocken 2015; Mohammadreza Shirzadi, Tominaga, and Mirzaei 2019a; S Murakami 1991), and computational fluid dynamics (CFD) (T van Hooff, Blocken, and Tominaga 2017; M. Shirzadi, Mirzaei, and Naghashzadegan 2018; Twan van Hooff, Blocken, and Tominaga 2016; 
Mohammadreza Shirzadi, Tominaga, and Mirzaei 2020; Ramponi and Blocken 2012a; M. Shirzadi, Naghashzadegan, and A. Mirzaei 2018; James Lo, Banks, and Novoselac 2013; Kosutova et al. 2019; Perén, Van Hooff, et al. 2015; Lo 2011; Hua, Ohbab, and Yoshiec 2006; Bazdidi-Tehrani et al. 2019).

Analyses of cross-ventilation flow over simplified isolated buildings has been studied extensively during the past thirty years (Etheridge 2015; Kato et al. 1992; Straw, Baker, and Robertson 2000; Jiang and Chen 2003; Ohba et al. 2004; Kobayashi et al. 2009, 2010; Ramponi and Blocken 2012b). The complex mechanism of cross-ventilation in mean flow structure as well as highly transient nature for an unsheltered cuboid building are discussed using extensive wind tunnel experiments by (Tominaga and Blocken 2016). On this basis, the presence of flapping jet and kelvin-Helmholtz instability around the windward jet was clearly shown using a special flow visualization technique (Tominaga and Blocken 2016; Mohammadreza Shirzadi, Tominaga, and Mirzaei 2020). Furthermore, application of CFD models, based on steady Reynolds-averaged Navier-Stokes equations (SRANS) model (T Kurabuchi et al. 2000; Straw, Baker, and Robertson 2000; Hu, Kurabuchi, and Ohba 2005; Kobayashi et al. 2010; Nikas, Nikolopoulos, and Nikolopoulos 2010; Perén, van Hooff, et al. 2015; M. Shirzadi, Mirzaei, and Naghashzadegan 2018; Mohammadreza Shirzadi, Mirzaei, and Naghashzadegan 2018) and large eddy simulation (LES) (Kato et al. 1992; T Kurabuchi et al. 2000; Akamine et al. 2004; Jiang et al. 2003; Hu, Ohba, and Yoshie 2008; Twan van Hooff, Blocken, and Tominaga 2016) were shown to be accurate enough for calculation of mean flow structure, mean turbulent statistics, crossing airflow rate, and surface pressure for cross-ventilation in unsheltered buildings.

In contrast to isolated building cases, fewer experimental and numerical studies are available related to the cross-ventilation in sheltered building conditions, which are realistic representation of buildings in urban areas. In an examples of in situ full-scale measurements by (H. L. Gough et al. 2018; H. Gough et al. 2018) over a group of buildings with planar density of $25 \%, 50 \%-90 \%$ airflow reduction was shown for a staggered arrangement of buildings in comparison with an isolated building. As another example of extensive wind tunnel experiments by (Tominaga and Blocken 2015; Mohammadreza Shirzadi, Tominaga, and Mirzaei 2019c, 2019b), 40\% reduction in the crossing airflow rate was observed using the tracer gas method over a group of generic buildings arranged in a regular configuration. In this study, velocity measurements by using split fiber probes (SPF) and flow visualizations revealed the complexity of flow structure of cross-ventilation in urban areas. In addition to the clear presence of highly transient flow features such as flapping jet and Kelvin-Helmholtz instability, the interaction of outside flow in the upstream and downstream cavity around the building and internal flow inside the cross-ventilated building was observed. Particle image velocimetry (PIV) was frequently used in wind tunnel measurements to aid the flow visualization. For example, (Ikegaya et al., 2019) observed a noticeable intermittency of cross-ventilation flow over a urban blocks with different arrangements while the effect of urban blocks arrangements on the mean flow structure and cross-ventilation statistics were studied.

CFD analysis of cross-ventilation for sheltered buildings is more challenging than the isolated building case. Despite the successful application of LES for cross-ventilation flow in isolated buildings, its application on cross-ventilation in urban areas is found to be difficult as much larger computational domain and more complicated geometries are necessary, which itself requires more computational cells, demanding more computational resources in comparison with the isolated building case. Example is CFD simulations by (King et al. 2017) using LES and lattice-Boltzmann method showed the effect of vortex shedding of upstream buildings on pulsating ventilation over a group of simplified buildings. They also 
reported the effect of building height on street canyon downdraft and velocity fluctuations around the cross-ventilated building.

In spite of proven acceptable accuracy of SRANS in cross-ventilation study for an isolated building, comparison of SRANS with experimental results surprisingly showed their poor accuracies in crossventilation prediction for sheltered buildings. For example, in a previous study by the authors where a group of buildings were arranged in a regular form with urban density of $25 \%$, SRANS models failed to reproduce the windward jet around the windward opening while deviations of up to $100 \%$ were found in the prediction of the crossing airflow rate and surface pressure difference over the building walls (Mohammadreza Shirzadi, Tominaga, and Mirzaei 2020; M. Shirzadi et al. 2018). Incapability of SRANS in calculation of transient flow features such as large-scale velocity fluctuations (Tominaga 2015) and uncertainty of the closure coefficients of turbulence models in SRANS (M. Shirzadi, Mirzaei, and Naghashzadegan 2018, 2017) are known as the main reasons behind such inaccuracy. Calibration of the closure coefficients of turbulence models was shown to be effective to improve the accuracy of SRANS for cross-ventilation modeling in urban areas (M. Shirzadi, Naghashzadegan, and A. Mirzaei 2018; M. Shirzadi et al. 2018; Mohammadreza Shirzadi, Naghashzadegan, and Mirzaei 2019).

An overview of the previous studies shows that more detailed studies are necessary for crossventilation flow in a sheltered building to understand 1) the dynamics of turbulent boundary layer above the buildings, within the street canopy, and inside the cross-ventilated building, 2) the interaction of turbulent flows within these three locations, 3) the contribution of transient flow characteristics on the mean flow structure, and 4) detailed analysis of turbulence modeling deficiencies in SRANS. On one hand, these finding are necessary for further turbulence model development. On the other hand, they help to better understand the limitation of numerical models for simulations of complex internal/external flows in urban studies. Thus, this study aims to address the above issues by using a series of wind tunnel experiments and CFD simulations using LES and SRANS for a cross-ventilation flow in a group of generic buildings, which are arranged in a regular configuration with a planar density ration of $25 \%$.

The novelty of this research is to conduct a detailed analyses by comparing SRANS and LES models to understand the physics of cross-ventilation in a sheltered building in terms of anisotropy turbulence characteristics and transient nature of flow, which are understood to have significant effect on the mean flow statistics. The anisotropy turbulence characteristics and transient nature of flow were studied well in literature for outdoor flow in urban areas, but there are very few studies about the complex internal/external flows such as cross-ventilation flow in buildings. In particular, the flow field around cross-ventilated building in sheltered conditions contains complex interactions between street canyon and cross-ventilation flows, which are dealing with more complexities comparing to the previous studies on external flows around buildings using LES and RANS models. Poor performance of SRANS in prediction of mean flow distribution in cross-ventilated buildings was shown in previous work by authors (Mohammadreza Shirzadi, Tominaga, and Mirzaei 2020; M. Shirzadi et al. 2018); but, in current research, LES is conducted for the first time over the wind tunnel experimental data, which was generated for the first time by the authors. Another novel aspect of this study is comprehensive validation and analysis of CFD models in which various factors, including mean velocity, turbulent fluctuation, pressure coefficient, crossing airflow rate, as well as flow visualization of transient flow are compared with wind tunnel experiments while their interactions on the cross-ventilation flow in sheltered condition were studied in detail. These make the current research unique as available studies in the literature generally focused on 
the physics of flow field, distribution of surface pressure, and crossing airflow rate separately, while all these parameters are considered in this study simultaneously.

In Section 2 and 3, details of the wind tunnel experiment and numerical settings for SRANS and LES are described. In Section 4, general flow field and accuracy of SRANS and LES are discussed. The turbulence statistics and transient flow property of cross-ventilation flow are presented in Section 5 and 6, respectively.

\section{Outlines of wind tunnel experiments}

The experimental measurements were conducted in the atmospheric boundary layer wind tunnel at Niigata Institute of Technology (Akabayashi et al. 1996; Kubota et al. 2008; Tominaga and Stathopoulos 2011; Yoshie et al. 2007). A neutral atmospheric boundary layer was created along the test section using spire and wooden roughness elements, which resulted to an aerodynamic roughness of $z_{o}=0.00033 \mathrm{~m}$. The target building was a cuboid with dimensions of $0.2 \mathrm{~m} \times 0.2 \mathrm{~m} \times 0.16 \mathrm{~m}$ for the building width, depth, and height $(W \times D \times H)$, with two openings of dimensions $0.036 \mathrm{~m} \times 0.092 \mathrm{~m}$ over the windward and leeward façades as shown in Figure 1(a). The target building was surrounded with eight similar buildings without opening, which were arranged in a regular configuration with a planar are ratio of $25 \%$. The building dimensions and configuration are the same as wind tunnel measurements conducted by (Tominaga and Blocken 2015), but the measurements are independently conducted. The vertical profiles of the time-averaged streamwise velocity $(U)$ and turbulent kinetic energy $(k)$ at the center of the empty turntable is shown in Figure 1(b). The approaching flow follows a power law with an exponent of $\alpha=0.25$ :

$$
\frac{U}{U_{H}}=\left(\frac{Z}{H}\right)^{0.25}
$$

where $U_{H}=5.233 \mathrm{~m} / \mathrm{s}$ is the time-averaged streamwise velocity at the reference height $H=0.16 \mathrm{~m}$. Instantaneous velocity components $(u, v, w)$ in the streamwise, lateral and vertical coordinates $(x, y, z)$ were measured over 119 measurement points using a split fiber probe (SPF) (Dantec Dynamics; 55R55) and a constant temperature anemometry module (Dantec Dynamics; $90 \mathrm{C10}$ ) by rotating the probe in corresponding direction. Moreover, the local wind surface pressure over 11 points was measured using a multi-point pressure transducer (Kyowa Electronic Instruments; F94-2206) (Tominaga et al. 2015). Furthermore, the crossing airflow rate through the target building were measured using a tracer gas method by injecting Ethylene near the windward façade while the concentration was simultaneously measured near the leeward opening with a high-speed total hydrocarbon analyzer (Technica, HTHCA-01). More details of measurements techniques, uncertainty, sampling rate and duration can be found in (Tominaga and Blocken 2015; Mohammadreza Shirzadi, Tominaga, and Mirzaei 2019c, 2019b). 


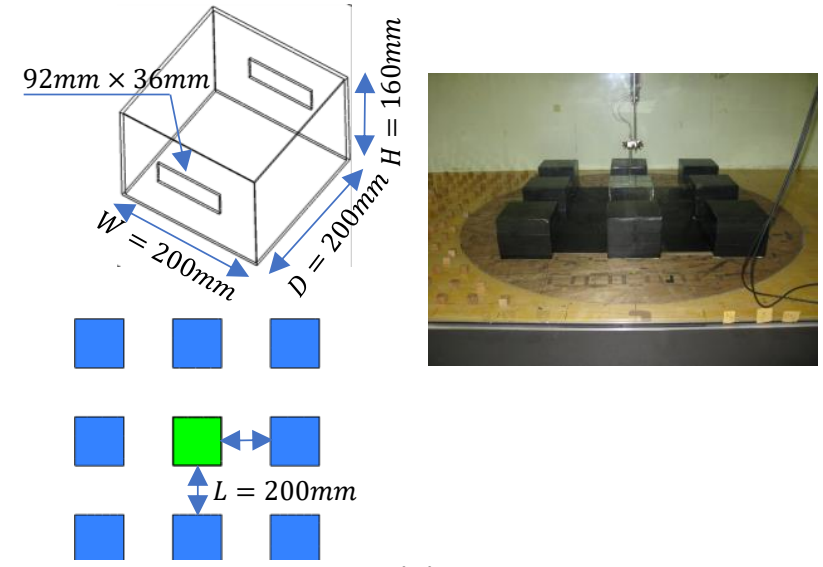

(a)

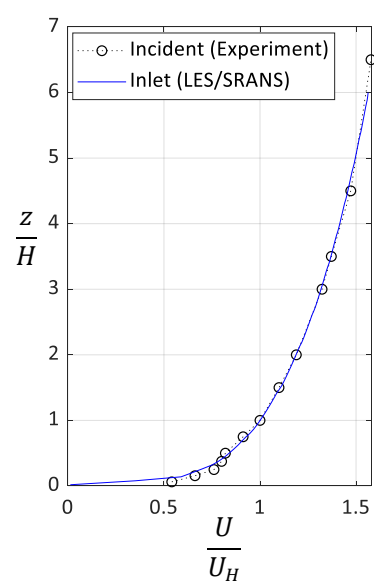

(b)

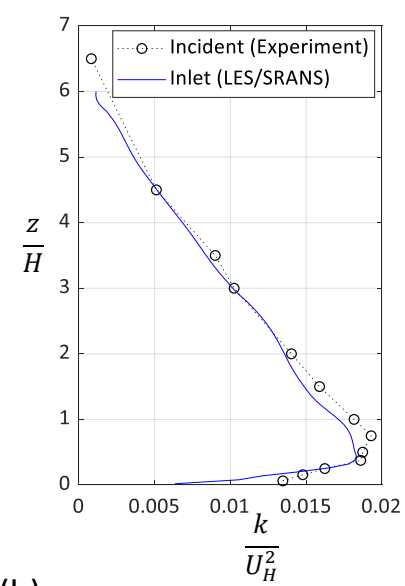

b)

Figure 1 (a) Building's dimensions and configuration, and (b) inlet and incident vertical profiles of $U$ and $k$

\section{Outlines of CFD simulation setup}

\subsection{Computational domain and grid}

A rectangular computational domain were created, which is extended to the wind tunnel sidewalls, as displayed in Figure 2 (Tominaga, Mochida, Yoshie, et al. 2008). The cut-cell method (lousef et al. 2017; Guichard 2019) was utilized to create three different grid settings as coarse, medium, and fine, which have $1,243,534,2,554,030$, and 4,758,001 cells, respectively. The size of edge elements over the opening, target building surfaces, surrounding building surfaces, and volume cell around all buildings is $0.002 \mathrm{~m}, 0.005 \mathrm{~m}$, $0.007 \mathrm{~m}$, and $0.007 \mathrm{~m}$ for the medium grid, respectively. For the coarse grid, the sizes of surface elements over the surrounding buildings walls and volume cell around all buildings are $0.01 \mathrm{~m}$ while these sizes are $0.005 \mathrm{~m}$ and $0.007 \mathrm{~m}$ for the fine grid. Boundary layer grid adjacent to the solid walls consists of five layers with the first layer height of $0.0001 \mathrm{~m}$ and an expansion ratio of $1.1 \mathrm{for}$ all grid settings. The average value of the non-dimensional wall distance over the building surfaces $\left(Y^{+}\right)$is less than 4 . A cross-section of the cell elements configurations over a vertical and horizontal plane for the medium grid are shown in Figure 2. The same computational domain and mesh were used for SRANS and LES.

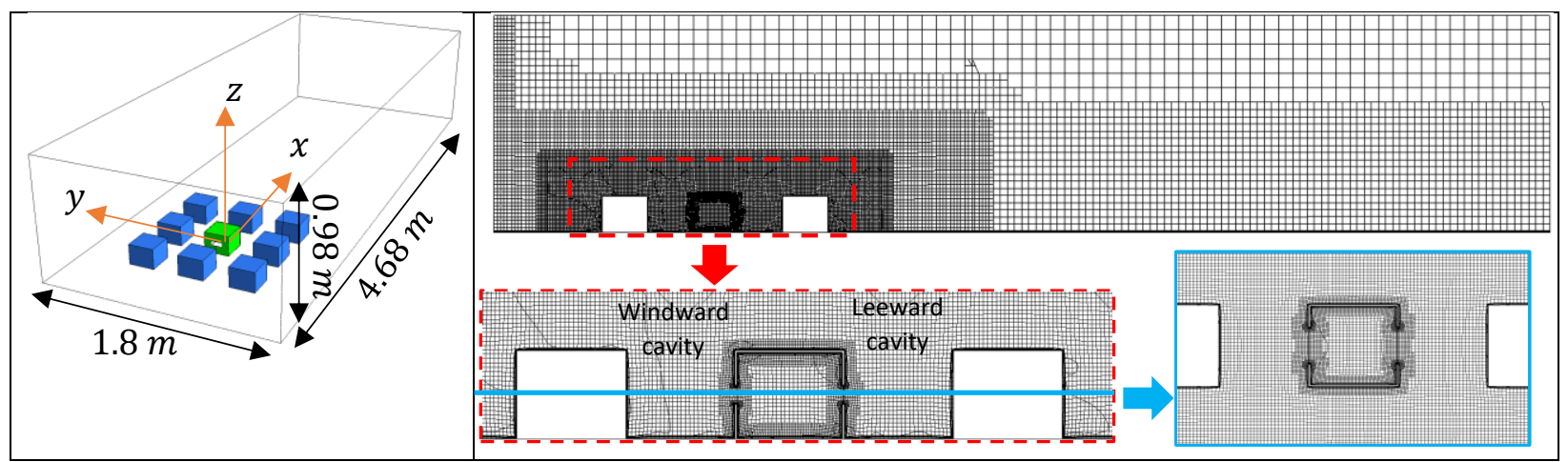

Figure 2 CFD computational domain and grid for medium grid

\subsection{Boundary conditions}

The experimentally measured incident vertical profiles of $U$ and $k$ were implemented directly as the inlet boundary condition for LES as shown in Figure 1(b). Velocity fluctuations at the inlet boundary were calculated using the Vortex method with 1,000 vortices (Sergent 2002). The obtained profiles for $U$ and $k$ 
at the inlet boundary by LES were implemented as the inlet boundary condition for SRANS in order to minimize the effect of different boundary conditions on the flow structure for more reliable comparison of turbulence models (Shirasawa et al. 2006; Shuzo Murakami et al. 1996). For SRANS, the inlet boundary condition for the turbulence dissipation rate $(\varepsilon)$ was obtained using the following equation by assuming a local equilibrium of the production of $k$ and $\varepsilon$ (Tominaga, Mochida, Yoshie, et al. 2008):

$$
\varepsilon(z)=\sqrt{0.09} k(z) \frac{U_{H}}{H} \alpha\left(\frac{Z}{H}\right)^{\alpha-1}
$$

Smooth wall boundary condition was assumed for the wind tunnel sidewalls and ground as well as all building walls while symmetry boundary condition was considered for the top boundary. For the outlet plane, a constant static pressure was applied.

\subsection{Solver settings}

ANSYS Fluent 19 was used to solve the governing equations for mass and momentum using the finite volume technique. For SRANS simulations, the SIMPLE method was used for the pressure-velocity coupling while the second-order upwind scheme was utilized for the advection terms discretization. Moreover, four two-equation turbulence models were used, including the standard $k-\varepsilon$ (Launder and Spalding 1974), RNG $k-\varepsilon$ (Yakhot and Orszag 1986), realizable $k-\varepsilon$ (Shih et al. 1995), and $k-\omega$ SST (Menter 1994). Accuracy of these turbulence models (except for the realizable $k-\varepsilon$ model) and effects of the discretization scheme of the advection and diffusion terms were investigated by (Mohammadreza Shirzadi, Tominaga, and Mirzaei 2020) for the same building configuration using ANSYS CFX solver. Furthermore, in the previous study, the performance of conformal structured hexahedral grid and unstructured tetrahedral grid settings was investigated in (Mohammadreza Shirzadi, Tominaga, and Mirzaei 2020) while a non-conformal grid based on the cut-cell technique, was utilized in order to reduce the computational cost in the current study. A convergence level of $10^{-5}$, based on the scaled residual, was considered for the mass, momentum, and turbulence transport equations.

For LES, the pressure-velocity coupling was based on the PISO solver. The bounded central differencing scheme was utilized for the spatial discretization of the momentum equation while the pressure interpolation was set as the second-order scheme. The transient formulation was based on the second-order implicit scheme. Furthermore, the standard Smagorinsky model was used with the Smagorinsky constant 0.12 (Ikegaya et al., 2019; Okaze et al., 2017). A time step of $\Delta t=0.0005 s$ was considered to satisfy the Courant number $C_{0}<1$ for all grid settings. The same time step was considered for the coarse, medium, and fine grid settings because the cell size around the target building was chosen to be very similar for all grid settings. The maximum Courant number, which occurs around the edges, was calculated to be less than 2 for the all meshes, and less than 1 in the rest of the domain. The statistics were calculated for $t=45 \mathrm{~s}$ in real time, which corresponds to a non-dimensional time unit of $t^{*}=$

$\frac{t U_{H}}{H}=1,471$. The statistical convergence was checked by monitoring the development of the timeaveraged velocity at several locations inside the building and street canyon.

\section{Results}

\subsection{Grid sensitivity analysis}

In Figure 3, the vertical profiles of $U$ calculated by LES are depicted for the different grid settings, which are found to be very close for medium and fine grid settings. The same results are obtained for the 
vertical profiles of $k$, but they are not shown due to the space limit. The ratio of the resolved kinetic energy to the total kinetic energy was estimated using the index of quality (IQ) for LES (Celik, Cehreli, and Yavuz 2005) to obtain more confidence about the quality of the LES results. The averaged values of IQ over different vertical lines are shown in Table 1 and are calculated to be higher than 80\% over all lines IQ, which is a recommended value by (Pope 2001) and corresponds to the previous urban CFD simulations using LES (Gousseau, Blocken, and Van Heijst 2013; Jörg Franke 2010). The difference between the prediction results of $U$ and $k$ by the course and medium mesh settings is very small, but the medium mesh shows a better performance in comparison to the coarse mesh in term of LES IQ. As shown in Table 1, for the coarse mesh, LES IQ over lines $\mathrm{J}$ and $\mathrm{K}$ is about 80 , which is very close to the commonly used thresholds in the literature. The LES IQs for the medium mesh over these two lines are $84 \%$ and $87 \%$, which are higher than those of the course mesh. Hence, for the rest of simulations, the medium grid setting is used for both LES and SRANS.

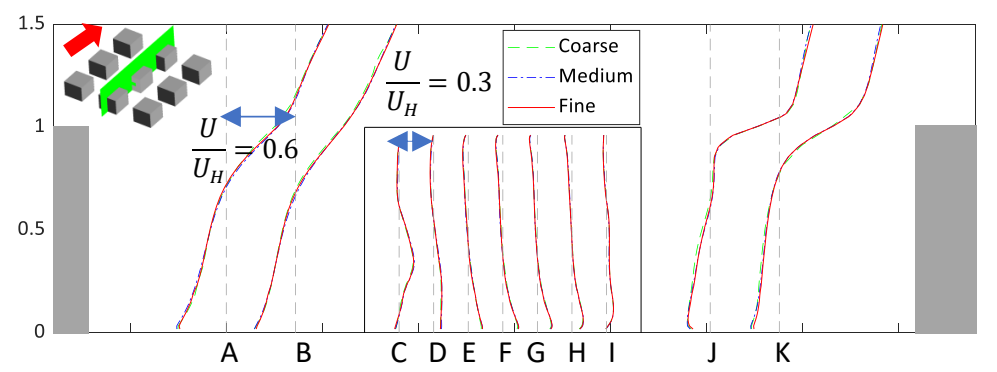

Table 1 LES quality index for different grid

\begin{tabular}{|l|l|l|l|l|l|l|l|}
\hline IQ (\%) & A & B & C & F & I & J & K \\
\hline Coarse & $\mathbf{8 4}$ & $\mathbf{9 1}$ & $\mathbf{9 0}$ & $\mathbf{9 2}$ & $\mathbf{8 6}$ & $\mathbf{8 1}$ & $\mathbf{8 0}$ \\
\hline Medium & $\mathbf{8 4}$ & $\mathbf{9 3}$ & $\mathbf{9 3}$ & $\mathbf{9 2}$ & $\mathbf{8 6}$ & $\mathbf{8 7}$ & $\mathbf{8 4}$ \\
\hline Fine & $\mathbf{8 5}$ & $\mathbf{9 2}$ & $\mathbf{9 1}$ & $\mathbf{9 3}$ & $\mathbf{8 7}$ & $\mathbf{8 5}$ & $\mathbf{8 5}$ \\
\hline
\end{tabular}

Figure 3 Vertical profiles of the time-averaged streamwise velocity

$U$ for different grid settings by LES

\subsection{Comparison of general flow field and validation metrics}

The distribution of $U$ over a vertical central plane and time-averaged streamlines are shown in Figure 4(a) for LES and SRANS with different turbulence models, i.e., the standard $k-\varepsilon(\operatorname{Std} k-\varepsilon), R N G k-\varepsilon$, Realizable $k-\varepsilon(\operatorname{Rel} k-\varepsilon)$, and $S S T k-\omega$ models. Above the street canyon, very similar velocity distributions are predicted by LES and all SRANS, whereas different flow distributions are obtained inside the street canyon and cross-ventilated building. LES and SST $k-\omega$ show large negative values caused by a strong reverse flow above the upstream building roof while no reverse flow is predicted by Std $k-\varepsilon$, $R N G k-\varepsilon$, and Rel $k-\varepsilon$. Furthermore, inside the upstream cavity (UC) and downstream cavity (DC), especially near the ground, negative values caused by the recirculation flows predicted by all SRANS to be much weaker that the one by LES. Comparison of the streamlines shows that different velocity distributions are obtained by all SRANS cases inside the building, which are found to be completely different from those by LES. Whilst a clear windward jet is observed through the windward opening in the streamwise direction by LES, none of the SRANS models could predict the windward jet. It is the most important feature of the cross-ventilation flow that is not predicted by SRANS. In contrary, a wide counterclockwise recirculation is predicted by the $S t d k-\varepsilon$ and $\operatorname{Rel} k-\varepsilon$ inside the building. In the case of the SST $k-\omega$ and $R N G k-\varepsilon$ models, the airflow direction is completely inverse inside the building where airflow enters the building through the leeward opening and exits through the windward one.

As depicted in Figure 4(b), whilst the predicted $k$ distributions by all SRANS and LES are generally similar above the canopy and upstream building's roof, noticeable differences can be observed inside the UC, DC, and building. In specific, the $k$ values predicted by all SRANS are much lower than those obtained 
by LES inside the UC and DC, which is also observed in an urban canopy simulation by (Nakajima, Ooka, and Kikumoto 2018). Despite a clear buildup of $k$ near the windward opening inside the building generated by LES, a uniform and relatively low $k$ distributions are estimated by all SRANS models. Details of these discrepancies will be analyzed in the following sections.

(a)
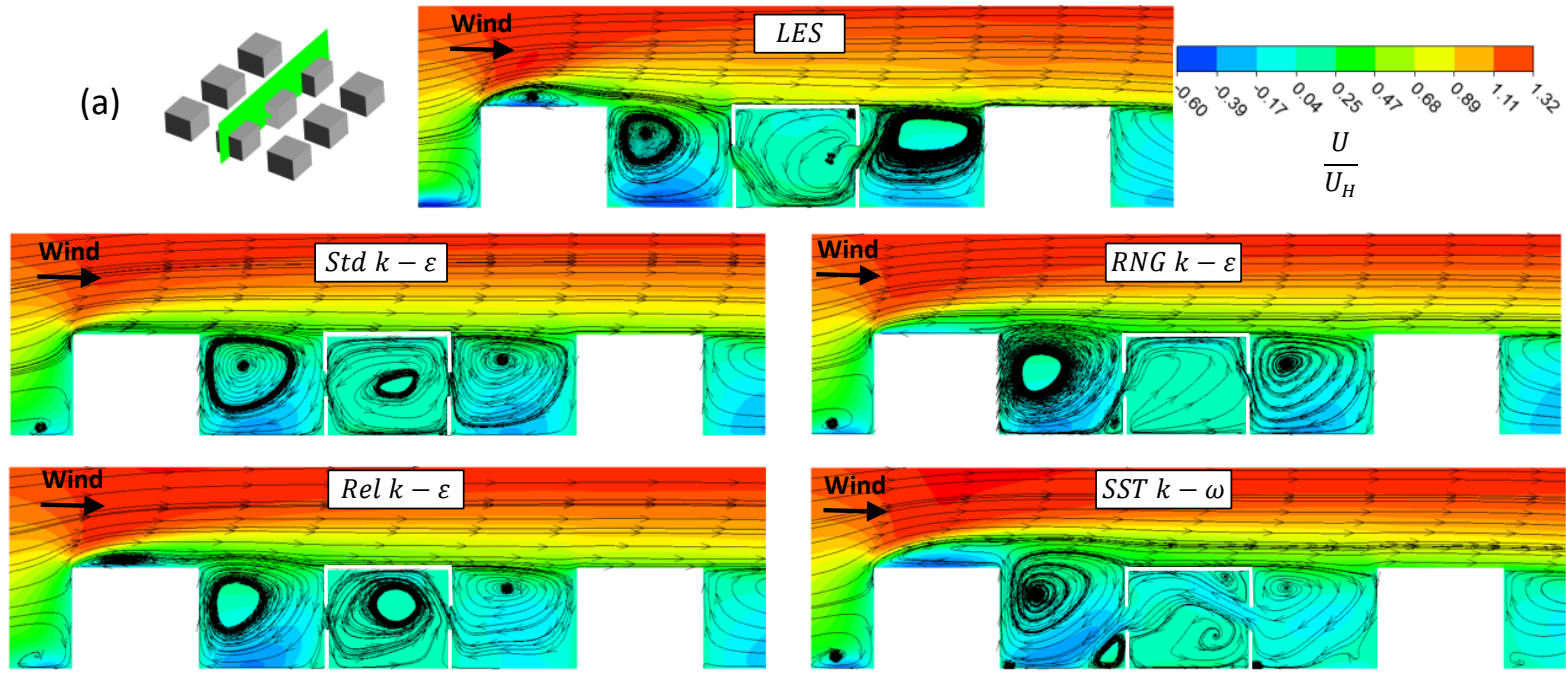

(b)
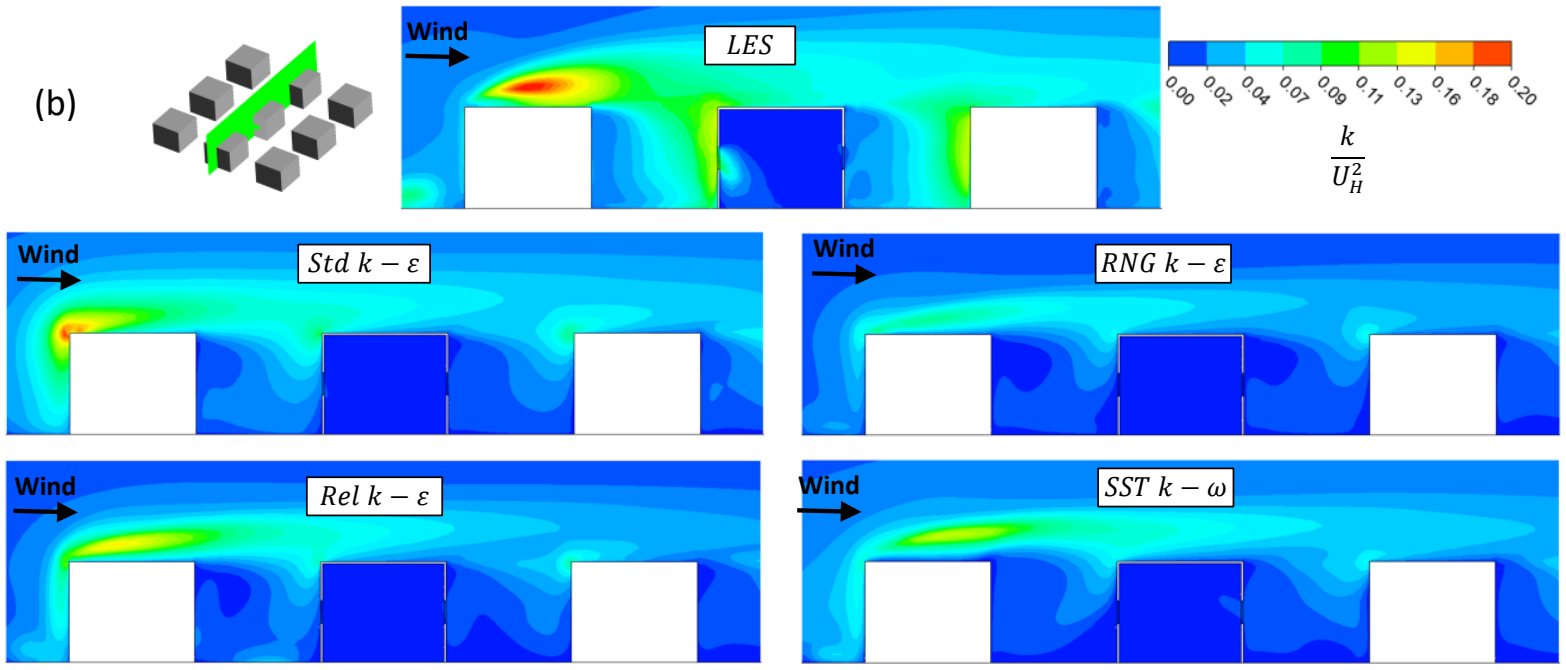

Figure 4 Distribution of (a) $U$ and (b) $k$ over a vertical central plane genrated by different turbulence models

The vertical profiles of $U$ and $k$ obtained by LES and all SRANS are compared against the experimental results in Figure 5. As shown in Figure 5(a), both LES and all SRANS predict very close values for $U$ over the vertical lines $A$ and $B$ inside the UC at the lower half of the cavity height $\left(0<\frac{z}{H}<0.5\right)$, whereas LES predictions are closer to the experiment at the upper half of the cavity height and above the roof $\left(0.5<\frac{z}{H}<1.5\right)$. The SST $k-\omega$ underestimates $U$ inside the UC and shows a different velocity profile in comparison to other SRANS. Inside the DC, the LES and all SRANS predictions are very close over Lines J and $\mathrm{K}$ at $0<\frac{Z}{H}<1$. Above the roof level $\left(1<\frac{Z}{H}<1.5\right)$, a good agreement between LES and the experiments can be observed, whilst all SRANS underpredict the velocity in this area. The accuracy of LES is shown to be noticeably higher than all SRANS in the prediction of the $U$ over Line $C$ near the windward opening inside the building, where the windward jet is accurately predicted by LES in comparison with the 
experimental measurements. In contrast, no windward jet is predicted by all SRANS as they are incorrectly found to be uniform inside the building, highlighting on a significant deviation from the experiment and LES. Whilst all SRANS predict a near zero value for $U$ inside the building, the $S S T k-\omega$ shows negative velocities over Lines $\mathrm{H}$ and $\mathrm{I}$, which wrongly implies on an entering air through the leeward opening. This is completely in an opposite direction of the cross-ventilation flow as observed in the experiment and LES.

As seen in Figure 5(b), LES and all SRANS calculations for $k$ are generally close to the experiment over Lines $A$ and $\mathrm{J}$ inside the UC and $\mathrm{DC}\left(0<\frac{Z}{H}<1\right)$. The overestimation of $k$ is observed above the cavity height $\left(1<\frac{z}{H}<1.5\right)$ along all lines by all SRANS and LES. Nonetheless, LES exhibits a very close agreement along Line $\mathrm{K}$ inside the DC while all SRANS show noticeable underpredictions. Inside the building, all SRANS results again show very inaccurate results while LES accurately predicts the profile around the windward jet (Line $C$ and $D$ ). The discrepancy between LES and experimental results over Line $B$ could be caused by the uncertainty of wind tunnel measurements near the windward façade where the presence of SPF and its supporting rod can distract airflow in that region. The same level of discrepancy is reported for a flow around an isolated building by (Ikegaya et al., 2019).

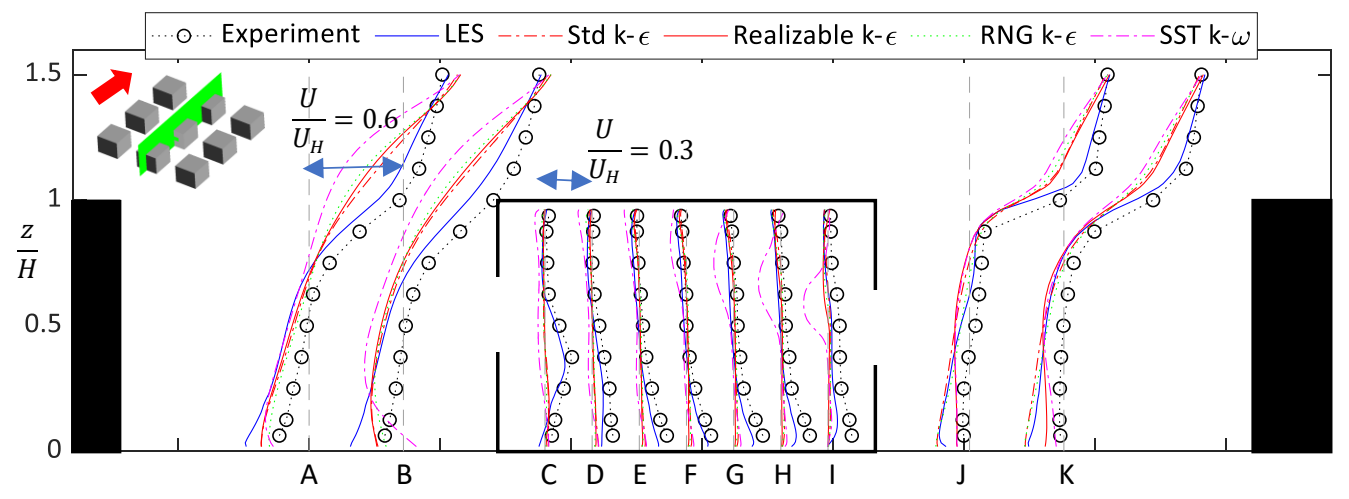

(a)

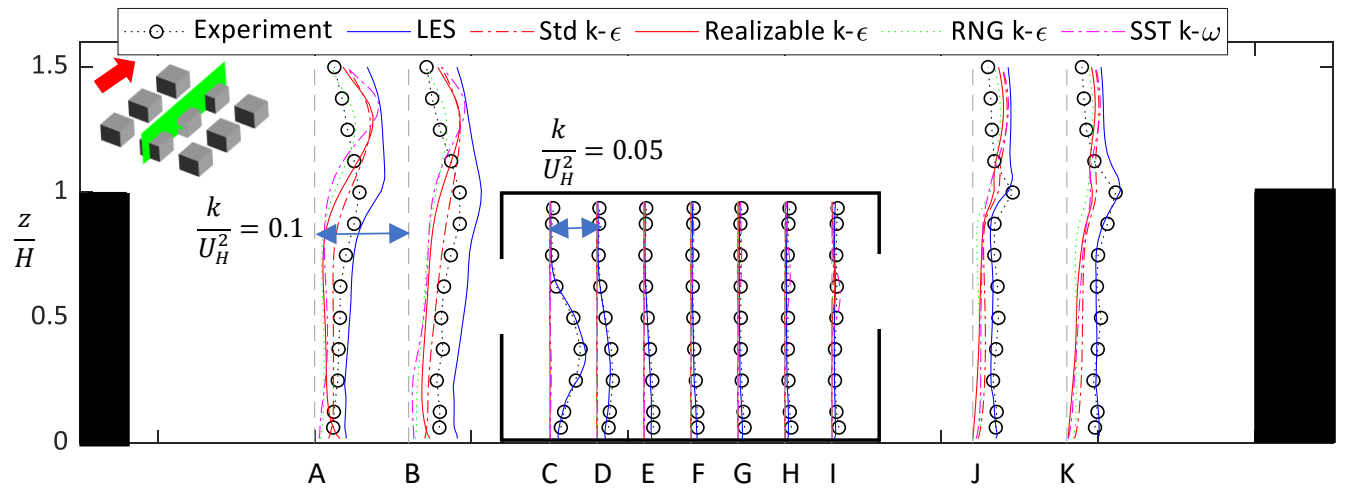

(b)

Figure 5 Vertical profiles of (a) $U$ and (b) $k$ genrated by LES and SRANS models

The accuracy of all SRANS and LES in prediction of time-averaged velocity components and $k$ is quantitatively assessed by validation metrics (Jörg Franke 2007; Schatzmann 2010), including the factor 
of two of observations ( $F A C 2)$, hit rate $(q)$, fractional bias $(F B)$, and normalized mean square error $(N M S E)$ :

$$
\begin{aligned}
& \text { FAC2 }=\frac{1}{n} \sum_{i=1}^{n} N_{i} \text { with } N_{i}=\left\{\begin{array}{l}
1 \text { for } 0.5 \leq \frac{P_{i}}{Q_{i}} \leq 2 \\
1 \text { for }\left|Q_{i}\right| \leq W \text { and }\left|P_{i}\right| \leq W \\
0 \text { otherwise }
\end{array}\right. \\
& q=\frac{1}{n} \sum_{i=1}^{n} N_{i} \text { with } N_{i}=\left\{\begin{array}{l}
1 \text { for }\left|\frac{P_{i}-Q_{i}}{Q_{i}}\right| \leq D \\
1 \text { for }\left|Q_{i}-P_{i}\right| \leq W \\
0 \text { otherwise }
\end{array}\right. \\
& F B=\frac{\langle Q\rangle-\langle P\rangle}{0.5(\langle Q\rangle+\langle P\rangle)} \\
& N M S E=\frac{\left\langle(P-Q)^{2}\right\rangle}{\langle Q\rangle\langle P\rangle}
\end{aligned}
$$

where $Q_{i}$ and $P_{i}$ are the observed (measured) and predicted (computed) values of a given variable, respectively, and $n$ is the number of data points. $D$ and $W$ are the thresholds for the allowed ranges. For $k, W=0.005$ while for the time-averaged velocity components, $W=0.05$ (Jorg Franke et al. 2011; Tominaga 2015). In FB and NMSE equations, the angular brackets stand for the average over all measurement points, which are calculated only for $k$.

As shown in Table 2, for LES, $F A C 2_{U}=0.58$, which is the highest values in comparison with the values obtained by $S t d k-\varepsilon, R N G k-\varepsilon$, Rel $k-\varepsilon$, and $S S T k-\omega$, which are $0.49,0.47,0.48$, and 0.33 , respectively. The same tendency is observed in prediction of the time-averaged vertical velocity $(W)$ where $F A C 2_{W}$ are $0.90,0.84,0.77,0.81$, and 0.70 respectively for LES, Std $k-\varepsilon, R N G k-\varepsilon$, Rel $k-\varepsilon$, and SST $k-\omega$. Surprisingly and despite a clear poor performance of all SRANS in the prediction of the main features of cross-ventilation (e.g. windward jet and internal velocity field as shown in Figure 4 and 5), the difference of the validation metrics between all SRANS and LES is not so large for the time-averaged velocity components. Therefore, assessment of CFD results performance based on these validation metrics should be performed carefully for the case of complex flows such as cross-ventilation in sheltered buildings.

The validation metrics for $k$ by LES are significantly better than those by SRANS. For LES, $F A C 2_{k}$, $q_{k}, F B_{k}$, and $N M S E_{k}$ are found to be0.90, 0.70, -0.31 , and 0.45 , respectively. These values for the $R N G k-\varepsilon$ and $R e l k-\varepsilon$ are respectively $0.42,0.38,0.77,1.48$, and $0.53,0.36,0.58,1.17$. The validation metrics for the SST $k-\omega$ is close to the those obtained by the RNG $k-\varepsilon$ and $\operatorname{Rel} k-\varepsilon$. Among all SRANS cases, the Std $k-\varepsilon$ shows the best agreement in the prediction of $k$ in comparison with the experimental results. For the $S t d k-\varepsilon, F A C 2_{k}=0.66$ and $q_{k}=0.41$ are the highest and $F B_{k}=0.35$ and $N M S E_{k}=0.61$ are the lowest values in comparison to the other SRANS results. The well-known overprediction of $k$ by the $S t d k-\varepsilon$ around the stagnation point, which is caused by the overestimation of the production term of $k$ (Murakami et al., 1990; Tominaga et al., 2008a; Tsuchiya et al., 1997), compensates the underprediction of $k$ inside the UC, DC, and building in SRANS; hence, for the Std $k-\varepsilon$, a relatively higher performance is observed in term of the validation metrics. 
Table 2 Validation metrics for LES and SRANS models

\begin{tabular}{|c|c|c|c|c|c|c|c|c|}
\hline & $F A C 2_{U}$ & $F A C 2_{W}$ & $F A C 2_{k}$ & $q_{U}$ & $q_{W}$ & $q_{k}$ & $F B_{k}$ & $N M S E_{k}$ \\
\hline Ideal values & 1 & 1 & 1 & 1 & 1 & 1 & 0 & 0 \\
\hline$L E S$ & 0.58 & 0.90 & 0.90 & 0.48 & 0.90 & 0.70 & -0.31 & 0.45 \\
\hline Std $k-\varepsilon$ & 0.49 & 0.84 & 0.66 & 0.41 & 0.80 & 0.41 & 0.35 & 0.61 \\
\hline RNG $k-\varepsilon$ & 0.47 & 0.77 & 0.42 & 0.43 & 0.70 & 0.38 & 0.77 & 1.48 \\
\hline Rel $k-\varepsilon$ & 0.48 & 0.81 & 0.53 & 0.41 & 0.77 & 0.36 & 0.58 & 1.17 \\
\hline SST $k-\omega$ & 0.33 & 0.70 & 0.44 & 0.21 & 0.66 & 0.35 & 0.55 & 1.34 \\
\hline
\end{tabular}

\subsection{Comparison of wind pressure and crossing airflow rate}

The profiles of the time-averaged wind surface pressure $\left(C_{P}=\frac{p-p_{r e f}}{\frac{1}{2} \rho U_{H}^{2}}\right)$ over the external and internal walls of the target building are depicted in Figure 6. Results of LES and all SRANS are compared against the wind tunnel measurements. Over the windward façade, the prediction values by all SRANS are noticeably lower than the LES and experimental results. For LES, $C_{P}$ peaks at two points around the stagnation point near the roof and near the ground as shown in Figure 6(a). For SRANS, the maximum $C_{P}$ over the windward façade is predicted by $S t d k-\varepsilon$, which is related to the overprediction of $k$ around the stagnation point. The profiles of $C_{P}$ obtained by the $R N G k-\varepsilon$ and $R e l k-\varepsilon$ are similar to that by the $S t d k-\varepsilon$ being almost negative over the windward façade. The $S S T k-\omega$ significantly underpredicts $C_{P}$ over the windward façade, which is caused by the strong reverse flow in the UC as seen in Figure 4(a). On the building's roof, all SRANS results show almost flat distributions of $C_{P}$, which is related to the absence of the reverse flow as seen in Figure 4(b) and stated by (Murakami et al., 1996). All SRANS and LES results are generally close over the roof far from the windward corner. Over the leeward façade, the prediction values of $C_{P}$ by SRANS and LES are generally close between the roof and upper edge of the leeward opening. Over the leeward opening and rest of the leeward façade, a good agreement between LES and the experiment can be observed, but the Rel $k-\varepsilon$ and $S S T k-\omega$ demonstrate significant underestimations. The distribution of $C_{P}$ over the internal surfaces are also shown in Figure $6(\mathrm{~b})$ for all SRANS and LES. For all cases, the distributions of $C_{P}$ are uniform over the internal walls. While LES shows noticeably a good agreement with the experimental result over the sidewalls and ceiling while all SRANS underpredict $C_{P}$ over the internal walls. 

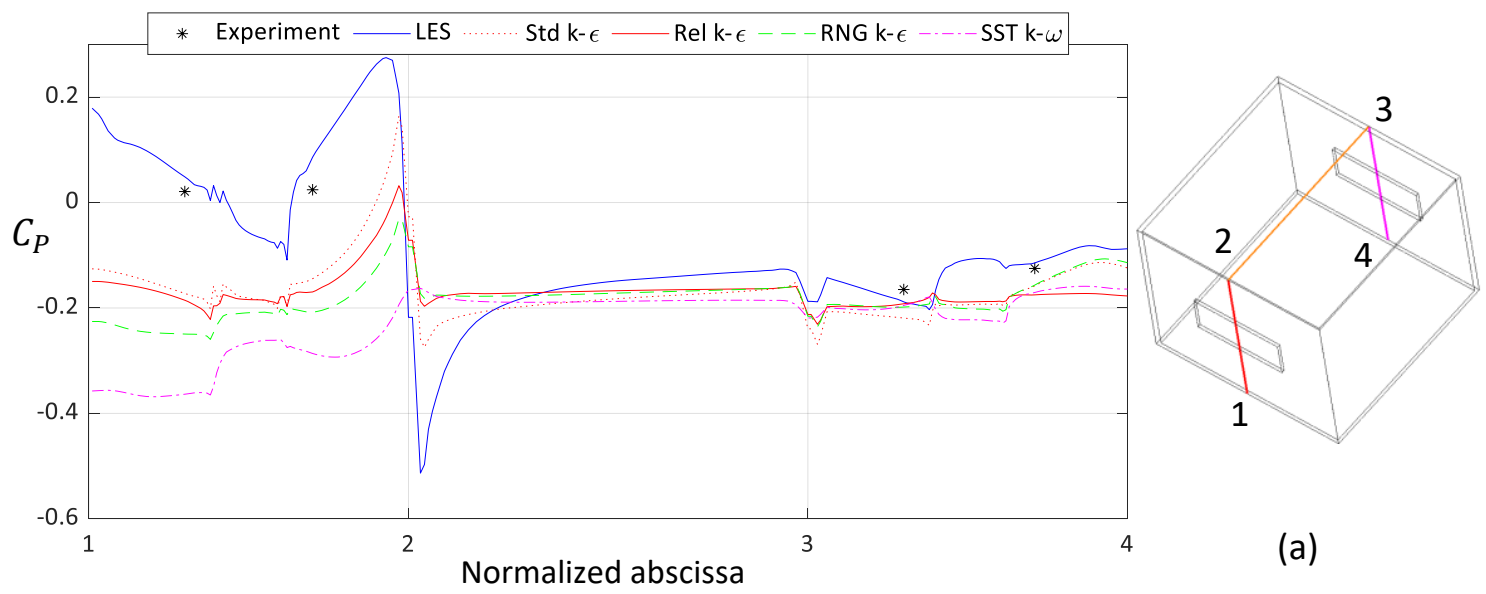

(a)
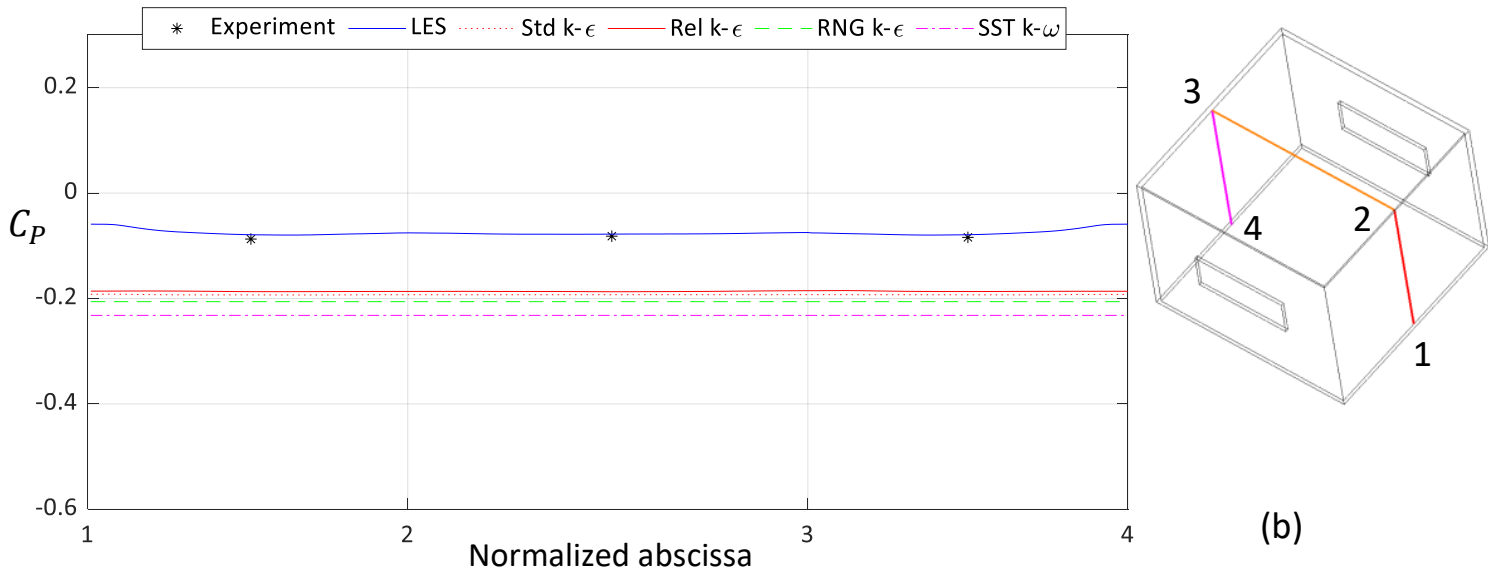

Figure 6 Profiles of the time averaged wind pressure over (a) external and (b) internal walls

The non-dimensional time-averaged crossing airflow rate $\left(\overline{q_{0}}\right)$, total wind surface pressure difference $\left(\Delta C_{p}^{\text {total }}\right)$, and windward wind surface pressure differences $\left(\Delta C_{p}^{w w}\right)$ are shown in Table 3 for all SRANS and LES, and compared with the experimental results. These quantities are defined as follows:

$$
\begin{aligned}
& \overline{q_{o}}=\frac{\bar{q}}{A U_{H}} \\
& \Delta C_{p}^{\text {total }}=\overline{C_{P}^{w w}}-\overline{C_{P}^{l w}} \\
& \Delta C_{p}^{w w}=\overline{C_{P}^{w w}}-\overline{C_{P}^{l n}}
\end{aligned}
$$

where $\bar{q}$ is the time-averaged volumetric airflow rate through the openings. $A$ is the opening area. $\overline{C_{P}^{w w}}$, $\overline{C_{P}^{l w}}$, and $\overline{C_{P}^{l n}}$ are respectively the average of wind surface pressure coefficient over the windward façades, leeward façades and interiors walls. The time-averaged volumetric airflow rate is calculated with the surface integration of the dot product of the time-averaged velocity vector $(\vec{V})$ and surface area vector $\overrightarrow{d A}$ over a virtual plane around the windward opening:

$$
\bar{q}=\iint \vec{V} \cdot \overrightarrow{d A}
$$

The experimentally measured crossing airflow rate is $\overline{q_{o}}=0.12$. As it can be expected from the velocity field, the LES prediction for $\overline{q_{o}}$ is very close to the experiment with a value of $\overline{q_{o}}=0.13$. In contrast, all SRANS results show very poor accuracy in prediction of the crossing airflow rate. The calculated crossing airflow rate by the Std $k-\varepsilon, R N G k-\varepsilon$, and $\operatorname{Rel} k-\varepsilon$ are around zero as no 
windward jet was predicted by these models (see Figure 5). A negative value of $\overline{q_{o}}(=-0.15)$ is calculated by the $S S T k-\omega$ model for the crossing airflow rate, which is also completely inaccurate.

The LES prediction of the wind surface pressure is very accurate in comparison to the all SRANS calculations. The LES results indicate $\Delta C_{p}^{\text {total }}=0.20$ and $\Delta C_{p}^{w w}=0.12$, which are in good agreement with the experiment $\left(\Delta C_{p}^{\text {total }}=0.17\right.$ and $\left.\Delta C_{p}^{w w}=0.1\right)$. In contrast to the experimental and LES results, highlighting on large pressure gradients across the cross-ventilated building, all SRANS results show a close to zero pressure gradient across the windward opening as well as the windward and leeward façades except for the SST $k-\omega$. For the Std $k-\varepsilon, R N G k-\varepsilon$, and $\operatorname{Rel} k-\varepsilon, C_{p}^{\text {total }}$ and $\Delta C_{p}^{w w}$ are found to be around zero, which are in agreement with a very low velocity inside the building as shown in Figure 5 . The SST $k-\omega$ results show a negative pressure difference over the building walls $\left(\Delta C_{p}^{\text {total }}=-0.15\right)$ and windward opening $\left(\Delta C_{p}^{w w}=-0.10\right)$. Thus, the negative crossing airflow rate is predicted by this model in accordance with the negative pressure difference as shown in Table 3.

Table 3 Crossing airflow rate and wind surface pressure
\begin{tabular}{|c|c|c|c|c|c|c|}
\hline & $E x p$ & $L E S$ & $\begin{array}{c}S t d \\
k-\varepsilon\end{array}$ & $\begin{array}{c}R N G \\
k-\varepsilon\end{array}$ & $\begin{array}{c}\text { Rel } \\
k-\varepsilon\end{array}$ & $\begin{array}{c}S S T \\
k-\omega\end{array}$ \\
\hline $\bar{q}$ & 0.12 & 0.13 & -0.02 & -0.05 & -0.03 & -0.15 \\
\hline$A U_{H}$ & & & & & & -0.15 \\
\hline$\Delta C_{P}^{\text {total }}$ & 0.17 & 0.20 & -0.01 & -0.06 & -0.02 & -0.15 \\
\hline$\Delta C_{P}^{w w}$ & 0.10 & 0.12 & 0.00 & -0.03 & -0.01 & -0.10 \\
\hline
\end{tabular}

\section{Detailed comparison between SRANS and LES}

It was shown that the performance of all SRANS are generally low in the prediction of the timeaveraged velocity, $k$, static pressure, and crossing airflow rate. More detailed comparison study is presented in the following section. For the brevity purpose, only the $S t d k-\varepsilon$ is considered as a turbulence model to represent SRANS family. Furthermore, as a two-equation turbulence model, the prediction accuracy of the $S t d k-\varepsilon$ was shown to be higher than other models in term of the validation metrics.

\subsection{Mean flow field}

The mean flow structure of the cross-ventilation is studied by comparing the time-averaged streamlines over a vertical central plane $\left(\frac{y}{H}=0\right)$ and a horizontal plane $\left(\frac{z}{H}=0.5\right)$ around the target building as shown in Figure 7. Also, the background is colored by the time-averaged velocity magnitude $(\overline{V m})$. LES shows a strong reverse flow over the upstream building roof while SRANS shows no reverse flow in this region. In LES, the reattached flow over the roof enters the UC with a larger inclined angle in comparison with SRANS in which the flow direction is parallel to the upstream roof and enters the UC with a small incident angle. The most obvious feature over the vertical plane in both models is a strong recirculation inside the UC. Associated with this vortex, a strong updraft near the leeward façade of the upstream building and strong downdraft near the windward façade of the target building are formed. The center of the recirculating vortex calculated by SRANS is almost at the same location as LES, but its vortex strength is weaker than LES. In LES, the strong downdraft along the windward façade partially enters through the windward opening, and then impinges toward the ground. The entering jet forms a counterclockwise recirculation flow and finally exits the building through the leeward façade with an 
upward orientation. In contrast, the SRANS result shows that the weak downdraft near the windward façade does not directly enter the buildings and mostly passes by the opening. As a result, the velocity magnitude inside the buildings is very small while the flow pattern is different from LES, which comprises of a large weak circulation inside the building. Inside the DC, LES shows a stronger reverse flow while the interaction between the leeward jet and the updraft flow inside the DC can be clearly observed near the leeward opening. The leeward jet pushes the recirculation center toward the streamwise direction and forms a small recirculation over the leeward opening, which cannot be observed in the SRANS result. The recirculation center predicted by SRANS is different from LES while no interaction is observed as no clear leeward jet is predicted by SRANS.

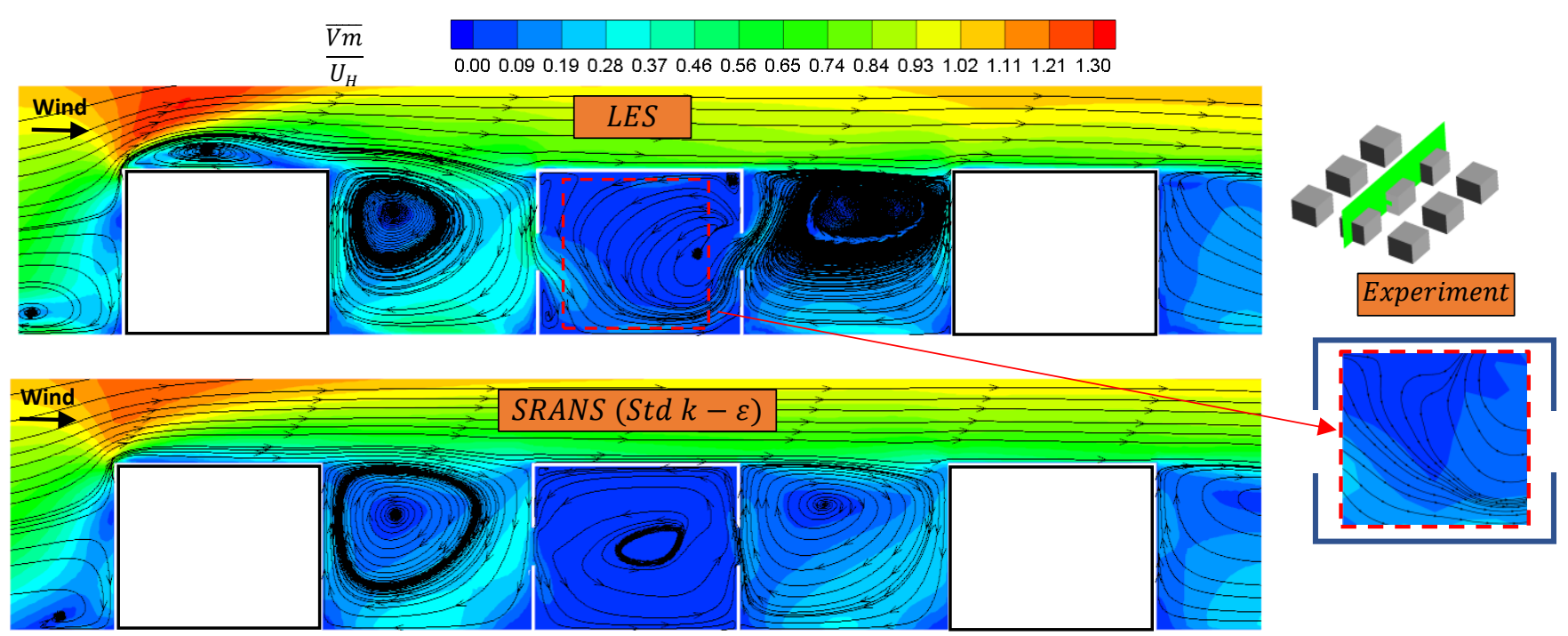

Figure 7 Time-averaged airflow streamlines coulured by the time-averaged velocity magnitude over a vertical central plane at $\frac{y}{H}=0$

The time-averaged streamlines over the horizontal plane at $\frac{Z}{H}=0.5$ are shown in Figure $8(\mathrm{a})$. A reverse flow is observed over the upstream building sidewall, which is captured by LES, but it is not predicted by SRANS. As a result, the lateral flow angle of the incoming air from the street canyon into the UC is oriented toward the UC center for LES, but for SRANS, the lateral flow angle is mostly parallel to the streamwise direction. The most evident difference is that the flow directions around the windward openings are completely opposite between LES and SRANS. The incoming flow from the outside UC partly enters to the windward opening and the rest of the flow forms a reverse flow in the cavity. The SRANS result also displays a weak recirculating vortex pair, which is stretched in the streamwise direction and creates a reverse flow; however, it does not have enough streamwise momentum to enter into the windward opening. The positions of the pair vortex centers are almost the same for SRANS and LES in the streamwise direction, but they are different in the lateral direction. Inside the building, LES clearly shows the streamlines of the entering flow through the windward opening and the leaving flow through the leeward opening while no crossing airflow is predicted by SRANS; the velocity magnitude inside the building is almost zero by SRANS. Inside the DC, a recirculating vortex pair is clearly predicted by LES, but the strength of the recirculating flow is rather weak as a result of the leeward jet, which pushes the vortex 
away from the leeward opening. The predicted recirculating vortex pair by SRANS is strong and very similar to the UC because there is no leeward jet to interact with the vortex pair.

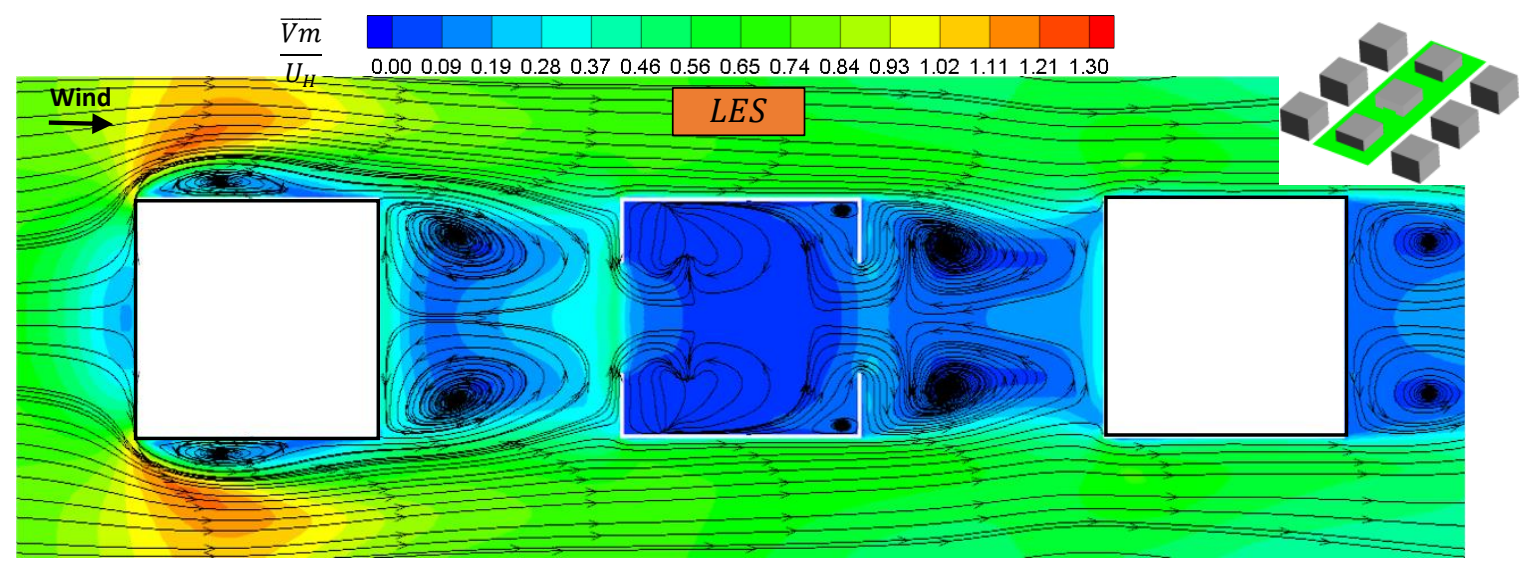

(a)

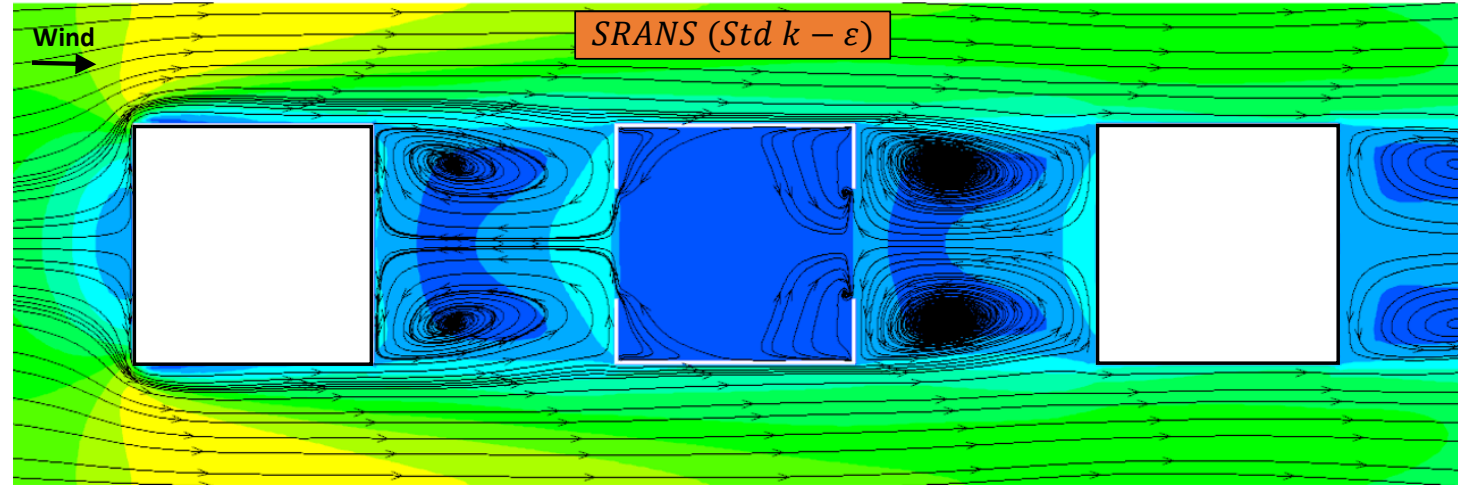

(b)

Figure 8 Time-averaged airflow streamlines coulured by velocity magnitude over a horizontal plane at $\frac{Z}{H}=0.5$

\subsection{Static pressure}

The distribution of the time-averaged wind pressure coefficient $\left(C_{P}\right)$ over the horizontal and vertical planes is shown in Figure 9 for LES and SRANS. In the vertical plane, LES shows large negative values of $C_{P}$ over the upstream building roof, which is associated to the presence of a roof separation and reattachment at this area as shown by the streamlines in Figure 7. The predicted negative $C_{P}$ by SRANS is much smaller than LES over the upstream building roof as no separation is calculated by SRANS. Similar distributions of $C_{P}$ are obtained by SRANS and LES near the upstream building wall inside the UC, but $C_{P}$ values by LES are much higher than SRANS around the windward façade. LES shows a very short separation around the roof edge of the target building as shown in Figure 6(a); nevertheless, no separation is predicted by SRANS, which results to the uniform $C_{P}$ distribution over the roof. A low-pressure region can be seen near the windward opening inside the building because the windward jet is clearly predicted by LES. As SRANS failed to calculate the windward jet, no pressure gradient can be observed inside the building. The similar pressure distributions are predicted by SRANS and LES inside the DC, however, the $C_{P}$ value by LES is higher than that by SRANS near the downstream building wall. Over the horizontal plane, as shown in Figure 9(b), LES shows negative values caused by the separation flows at the sidewalls and a clear $C_{P}$ buildup near the windward façade. The large $C_{P}$ are predicted in front of the windward façade in LES as well as the vertical plane. A uniform $C_{P}$ distribution is estimated inside the building by both models, 
but the values of LES are higher than that of SRANS. The uniform $C_{P}$ distribution is also predicted by SRANS inside the DC, which is at the same level as LES, except near the downstream building wall.

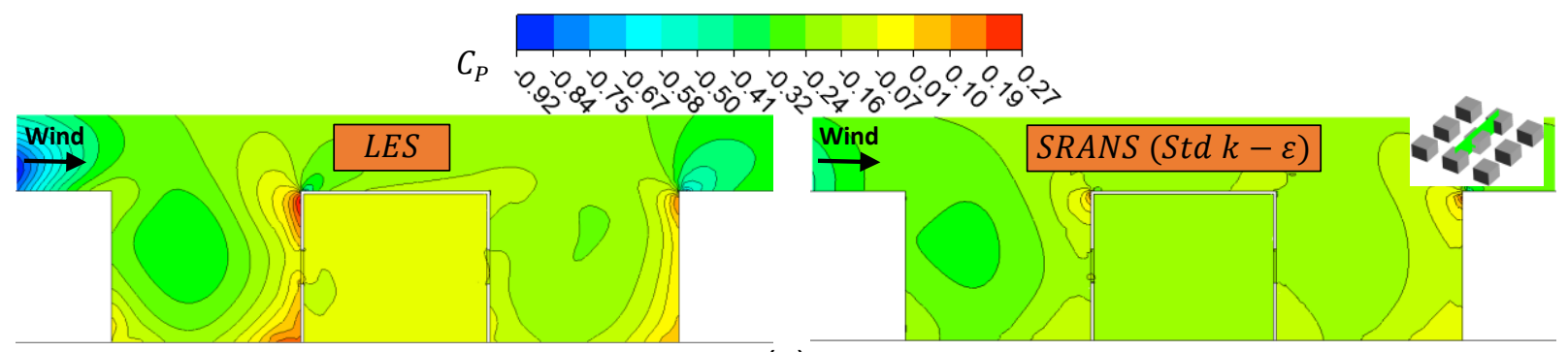

(a)
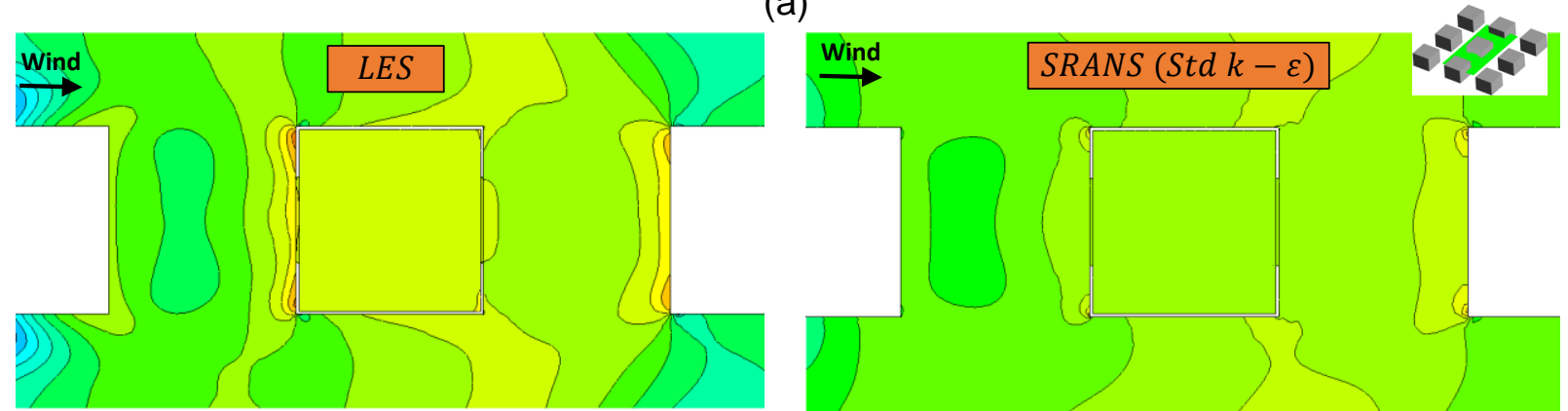

(b)

Figure 9 Distribution of the time-averaged wind surface pressure over $(a)$ vertical plane $\left(\frac{y}{H}=0\right)$ and $(b)$ horizontal plane $\left(\frac{z}{H}=0.5\right)$

\subsection{Turbulence statistics}

In order to understand more details about the deficiency of SRANS for the cross-ventilation flow under sheltered condition, the distributions of the turbulence statistics calculated by SRANS are compared with the results of LES and the experiment.

The distributions of three velocity fluctuations in the streamwise, lateral and vertical directions $\left(\left(\overline{u^{\prime} u^{\prime}}\right)^{1 / 2},\left(\overline{v^{\prime} v^{\prime}}\right)^{1 / 2},\left(\overline{w^{\prime} w^{\prime}}\right)^{1 / 2}\right)$ over the vertical central plane are shown in Figure 10 for LES and SRANS. The experimental results are displayed only inside the building. For SRANS, three components of the velocity fluctuations are calculated using the eddy viscosity model (Shuzo Murakami, Mochida, and Hayashi 1990; Tominaga, Mochida, Murakami, et al. 2008; Nakajima, Ooka, and Kikumoto 2018):

$$
\begin{aligned}
& \left(\overline{u^{\prime} u^{\prime}}\right)^{1 / 2}=\left(-2 v_{t} \frac{\partial U}{\partial x}+\frac{2}{3} k\right)^{1 / 2} \\
& \left(\overline{v^{\prime} v^{\prime}}\right)^{1 / 2}=\left(-2 v_{t} \frac{\partial V}{\partial y}+\frac{2}{3} k\right)^{1 / 2} \\
& \left(\overline{w^{\prime} w^{\prime}}\right)^{1 / 2}=\left(-2 v_{t} \frac{\partial W}{\partial z}+\frac{2}{3} k\right)^{1 / 2}
\end{aligned}
$$

According to the LES calculation, the velocity fluctuation in the streamwise direction, $\left(\overline{u^{\prime} u^{\prime}}\right)^{1 / 2}$ is very large above the upstream building roof with a maximum value of 0.5 , which means it is half of the timeaveraged mean velocity at the reference height. The value of $\left(\overline{u^{\prime} u^{\prime}}\right)^{1 / 2}$ decreases inside the UC and DC, but a clear growth can be seen around the windward jet with a maximum value of about 0.3. Interestingly, the velocity fluctuation in the lateral direction, $\left(\overline{v^{\prime} v^{\prime}}\right)^{1 / 2}$ is significantly higher than the streamwise and 
vertical components in front of the target building. In specific, the higher values of $\left(\overline{v^{\prime} v^{\prime}}\right)^{1 / 2}$ are predicted over the windward façades of the target and downstream buildings. This indicates a significant contribution of the vortex shedding flow and the lateral momentum transfer in the cavity on the crossventilation flow as the time-averaged lateral velocity is zero over the vertical plane (symmetric plane). The distribution of the vertical component $\left(\overline{w^{\prime} w^{\prime}}\right)^{1 / 2}$ is more uniform in comparison with the streamwise and lateral components and shows a same tendency around the windward jet as the streamwise component. The most obvious feature of the vertical component around the windward opening is its spread toward the ground in comparison with the streamwise and lateral components, which are generally spread in the streamwise direction.

Inside the building, LES results show very good agreement with the experimental measurements. A noticeable feature in the velocity fluctuations calculated by LES is the anisotropy of the turbulence inside the UC and DC, and building. In contrary, the velocity fluctuations by SRANS show an isotropic property as the distributions of each component are very similar mainly related to the isotropic eddy viscosity assumption. Although the prediction accuracy of $k$ in the UC in SRANS looks acceptable as shown in Figure 5(b), SRANS significantly underestimates the velocity fluctuations around the windward jet and inside the building, where the values of $\left(\overline{u^{\prime} u^{\prime}}\right)^{1 / 2},\left(\overline{v^{\prime} v^{\prime}}\right)^{1 / 2}$, and $\left(\overline{w^{\prime} w^{\prime}}\right)^{1 / 2}$ are almost zero.

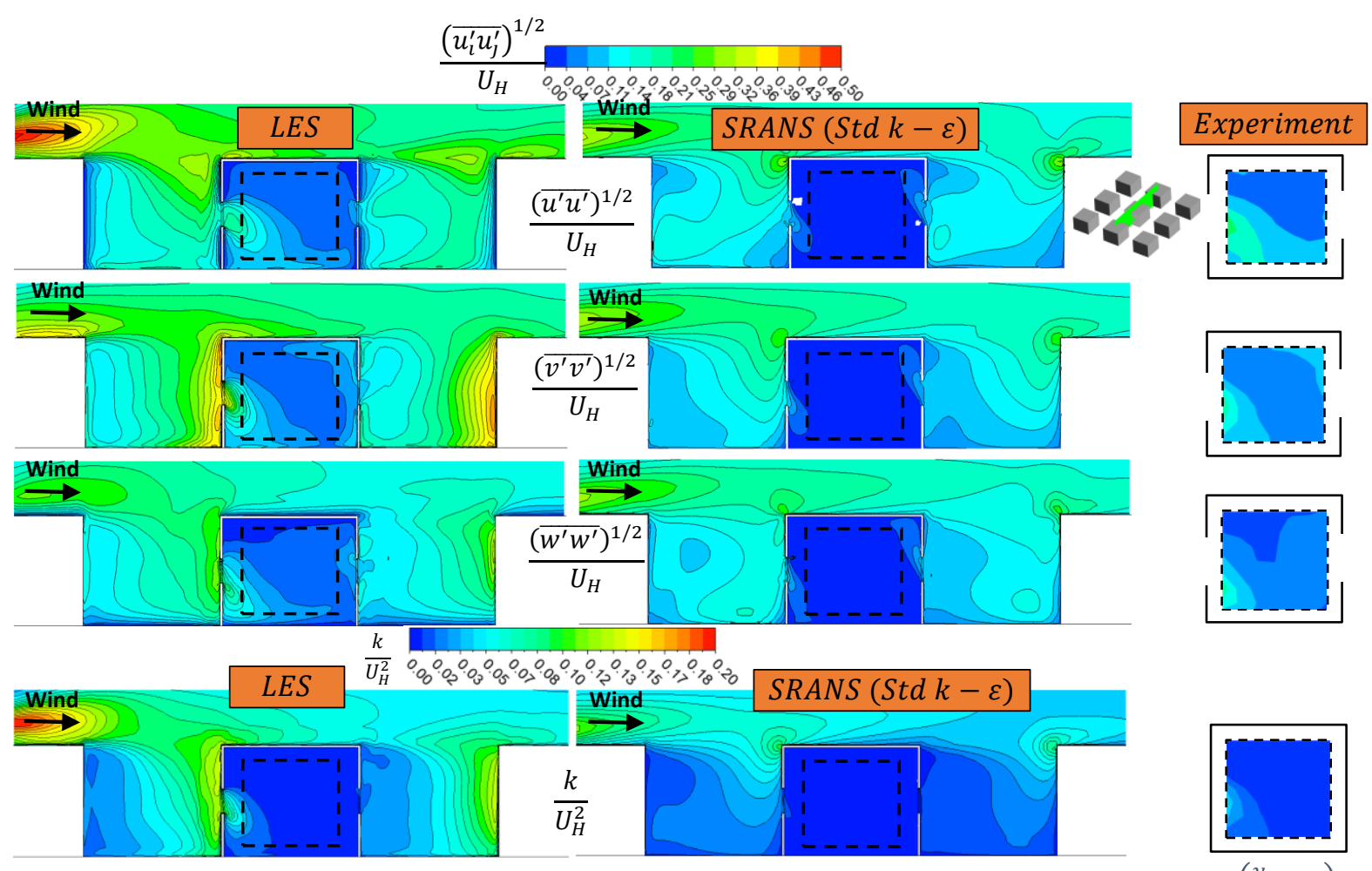

Figure 10 Distribution of the velocity fluctuation components and $k$ over a verical central plane $\left(\frac{y}{H}=0\right)$

The distributions of the velocity fluctuation components and $k$ over the horizontal plane $\left(\frac{z}{H}=0.5\right)$ are displayed in Figure 11 for LES and SRANS. The trend is similar to one discussed over the vertical central plane shown in Figure 10. For LES, values of $\left(\overline{u^{\prime} u^{\prime}}\right)^{1 / 2}$ are noticeably high around the upstream building sidewalls. Inside the UC, $\left(\overline{u^{\prime} u^{\prime}}\right)^{1 / 2}$ is very low behind the upstream building wall, but it rises significantly 
around the windward opening and inside the building around the windward jet. In the DC, $\left(\overline{u^{\prime} u^{\prime}}\right)^{1 / 2}$ shows a slight increase around the leeward opening due to the interaction of the leeward jet and reverse flow inside the UC. The higher values of the lateral component, $\left(\overline{v^{\prime} \bar{v}^{\prime}}\right)^{1 / 2}$ are obtained by LES near the windward jet inside the UC, indicating the effect of the lateral turbulent momentum transfer in this region. The same level of $\left(\overline{v^{\prime} v^{\prime}}\right)^{1 / 2}$ can be observed in the DC in front of the downstream building wall. Also, the vertical component $\left(\overline{w^{\prime} w^{\prime}}\right)^{1 / 2}$ peaks around the upstream building sidewalls and in front of the central and downstream buildings. Around the leeward jet inside the DC, all three components of the fluctuation velocity are calculated at the same level. Unlike LES, the SRANS results show very similar distributions for $\left(\overline{u^{\prime} u^{\prime}}\right)^{1 / 2},\left(\overline{v^{\prime} v^{\prime}}\right)^{1 / 2}$, and $\left(\overline{w^{\prime} w^{\prime}}\right)^{1 / 2}$. This indicates that the anisotropic property of the velocity fluctuations, which is clearly observed in the experiment and LES, cannot reproduced by SRANS. As already shown in Figure 4(b), the predicted value of $k$ by LES is significantly higher than that of SRANS inside the UC and around the windward jet inside the building. For SRANS, the value of $k$ is almost zero inside the building. 

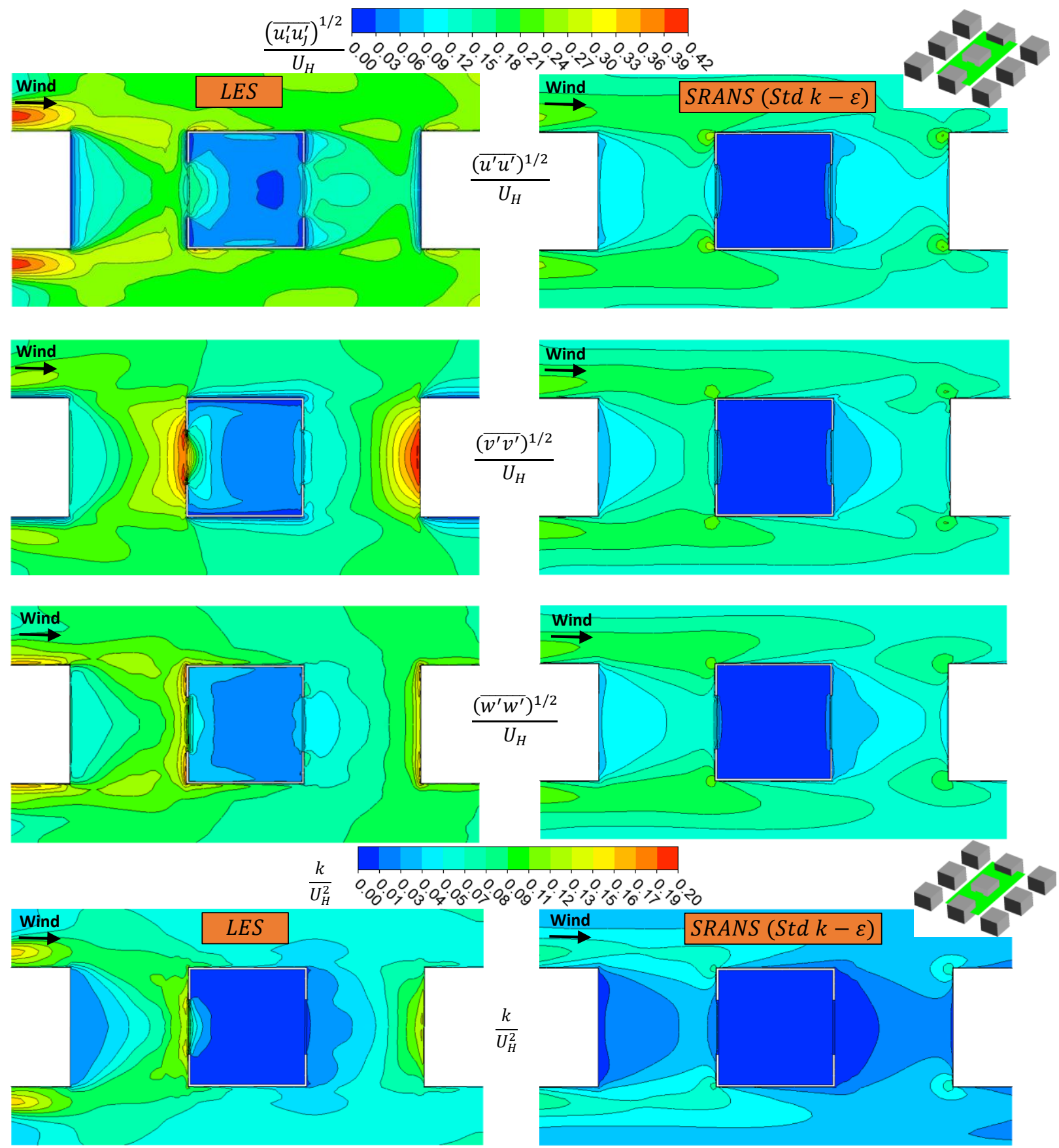

Figure 11 Distribution of the velocity fluctuation components and $k$ over a horizontal plane $\left(\frac{Z}{H}=0.5\right)$

\section{Unsteady flow visualization}

In the previous sections, the anisotropic turbulence property, which is observed in the experiment and LES, but not in SRANS, are displayed. In this section, the instantaneous snapshots of the flow field are further demonstrated to understand the nature of the transient flow, affecting the time-averaged flow structure. 


\subsection{Transient airflow rate}

The instantaneous non-dimensional crossing airflow rate through the windward opening $q_{o}=\frac{q}{A U_{H}}$ by LES is shown in Figure 12 for 150 non-dimensional time-steps from the total 1,417 non-dimensional sampling time-steps. The instantaneous airflow rate is calculated as $q=\iint \vec{V} \cdot \overrightarrow{d A}$ using the instantaneous velocity vectors $(\vec{V})$. While the time-averaged airflow rate by LES is found to be $\overline{q_{o}}=0.13$, the instantaneous airflow rate fluctuates significantly between $-0.03 \leq q_{o} \leq 0.26$. The maximum value of instantaneous airflow rate is almost twice the time-averaged value while its minimum value is about zero. The crossing airflow rate fluctuations are repeated with a frequency of about $1 / 30$. Moreover, four snapshots are selected over an airflow fluctuation cycle to investigate the instantaneous velocity field in the following discussion.

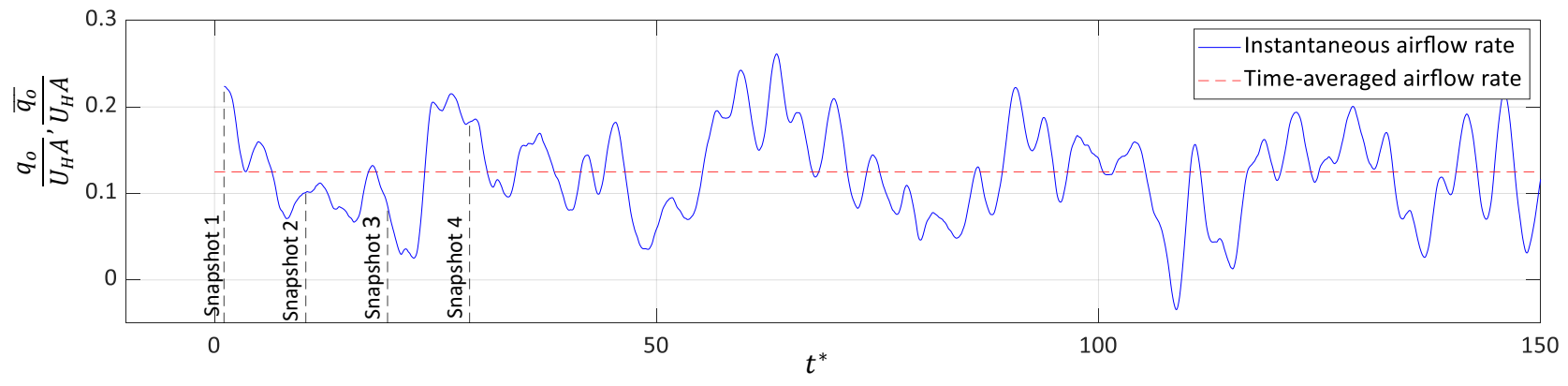

Figure 12 Instantnaous and mean crossing airflow calculated by LES

\subsection{Instantaneous velocity field}

The instantaneous snapshots of the velocity field by LES over the vertical plane $\left(\frac{y}{H}=0\right)$ are shown in Figure 13 at $t^{*}=0, t^{*}=9.27, t^{*}=18.53$, and $t^{*}=27.80$ with a constant non-dimensional time interval of $\Delta t^{*}=9.27$ (see Figure 12). The figure background is colored by the instantaneous velocity magnitude, but it is intentionally shown transparent in order to optimize the visibility of the velocity vectors. The experimental visualizations, which were conducted by using of a fog generator, a laser light sheet, and a high-speed camera are also shown only inside the target building. The instantaneous flow pattern is completely different with the time-averaged one shown in Figure 7. The presence of the vertical structures is observed clearly around the buildings. A glance at the wind vectors of all snapshots indicates a complicated distributions of the vortices inside the UC and DC. The turbulent momentum transfer in the vertical direction occurs by these vortices, which are known as sweep and injection ones (Coceal et al. 2006; Nakajima, Ooka, and Kikumoto 2018).

At the first snapshot $\left(t^{*}=0\right)$, when the instantaneous airflow rate is almost maximum $\left(q_{o}=0.22\right)$, a region of large streamwise velocity component with downward orientation can be seen above the UC center. A clockwise circulation is created under this region close to the upstream building wall inside the UC, which is in contact with the reverse flow at the mid-height of the UC. The velocity direction is upward around the windward façade of the central building, which pushes the flow above the roof and enhances the roof separation. The flow in front of the windward opening is also in the upward direction, but the flow partially penetrates into the building. A large circulation can be also observed inside the DC, which joins with the leeward jet crossing through the leeward opening. 
In the next time-step at $t^{*}=9.27$, which corresponds to the period with $q_{o}=0.10$, the high momentum airflow above the UC can be observed in the first snapshot, passing over the UC and creating a strong downdraft near the windward façade of the target building. As a result, the windward jet is clearly oriented toward the ground and a large circulation is generated inside the building. At the same time, a reverse flow is seen above the target building roof while a very weak downdraft is observed near the downstream building wall inside the DC, which forms a very complex flow pattern with a very low velocity distribution around the leeward opening.

At the $3^{\text {rd }}$ snapshot $\left(t^{*}=18.53\right)$, the airflow rate is very similar to the $2^{\text {nd }}$ snapshot with a value of $q_{o}=0.09$. Despite a very close crossing airflow rate, very different instantaneous velocity fields are observed in these two snapshots. Most of the airflow is in the horizontal direction above the UC, therefore, there is no updraft near windward façade of the target building at roof height inside the UC. In the absence of the strong updraft near the windward corner of the target building roof, the separation does not occur as a result of the high momentum flow in the streamwise direction above the canopy. Inside the building, the windward jet sweep forward on the ground and then moves downward to form a large counterclockwise circulation. The flow field inside the DC consists of a strong reverse flow near the downstream building wall and a weak upward flow near the leeward opening.

In the $4^{\text {th }}$ snapshot at $t^{*}=27.80$, the airflow rate increases again and reaches to $q_{o}=0.18$. The general flow field is nearly, but not completely, similar to the $1^{\text {st }}$ snapshot, featuring a region of the large streamwise velocity with downward orientation above the UC center and a large circulating flow inside the DC, which interacts with the leeward jet. Around the windward opening, the flow is in the horizontal direction, then forms a recirculation flow inside the building. 

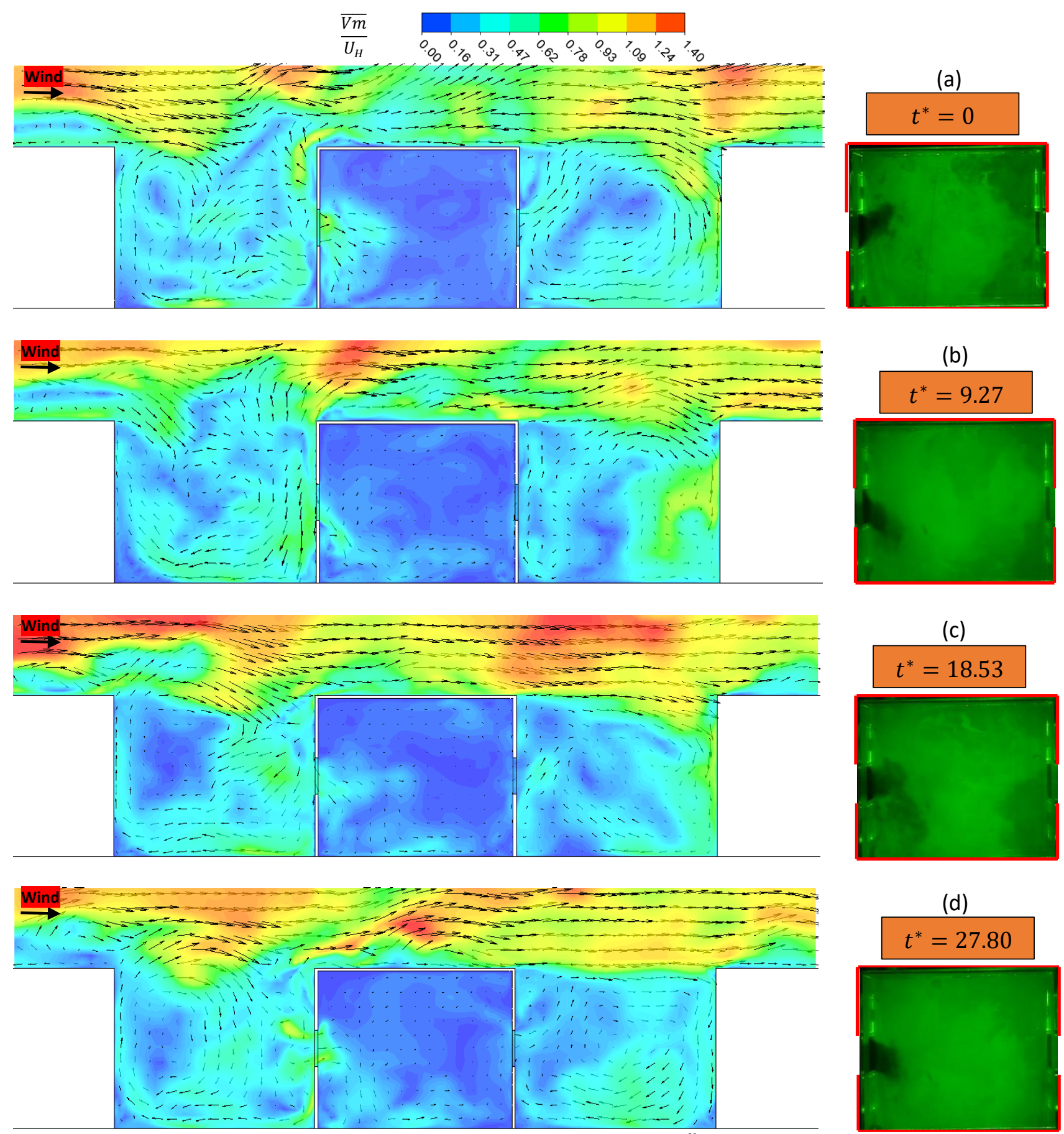

Figure 13 Instantaneous snapshots of the velocity field over a vertical plane $\left(\frac{y}{H}=0\right)$ at four different non-dimensional times

The instantaneous snapshots of the velocity field by LES over the horizontal plane $\left(\frac{Z}{H}=0.5\right)$ are shown in Figure 14 at the times similar to the vertical plane in Figure 13 . At the $1^{\text {st }}$ snapshot, a region with a high streamwise velocity is moving inside the UC from the left lateral street. This motion forms a strong windward jet through the windward opening with a same orientation of the approaching flow from the left lateral street. In the right side of the UC, a strong reverse flow reaches to the right lateral street flow. As a result, a circulation is formed near the right side of the windward façade. The leaving flow through 
the leeward opening is oriented toward the right lateral street where mixes with the entering flow from the right lateral street inside the DC.

For the $2^{\text {nd }}$ snapshot, a high velocity flow from the left lateral street hits the windward façade and forms a reverse flow. As a result, no windward jet near the windward opening can be observed over the horizontal plane. However, at the same time, the strong downdraft over the vertical plane, as shown in Figure 13(b), which has clearly more momentum in the vertical direction, creates a downward windward jet. Inside the DC, the flow from the right lateral street completely sweeps onto the downstream building wall and moves to the left lateral street.

In the $3^{\text {rd }}$ snapshot, the interaction of the approaching flows from the left and right lateral streets forms a narrow windward jet close to the left corner of the windward opening. The high momentum flows from the right and left lateral streets remove the sidewall separation over the central building sidewalls. Thus, a narrow leaving jet is formed around the right side of the leeward opening, which mixes with the incoming flow from the right lateral street. Then, it sweeps on the downstream building wall inside the DC and enters the left lateral street.

The flow field at the $4^{\text {th }}$ snapshot shows almost left and right reversed distribution of the one observed for the $1^{\text {st }}$ snapshot. In this condition, a high momentum flow enters the UC from the right lateral street and creates a strong windward jet toward the left side wall of the target building. The rest of the incoming flow mixes with the parallel street canyon flow. Inside the DC, the leaving flow through the leeward opening joins with the incoming flows from both street canyons and forms weak recirculation flows. 

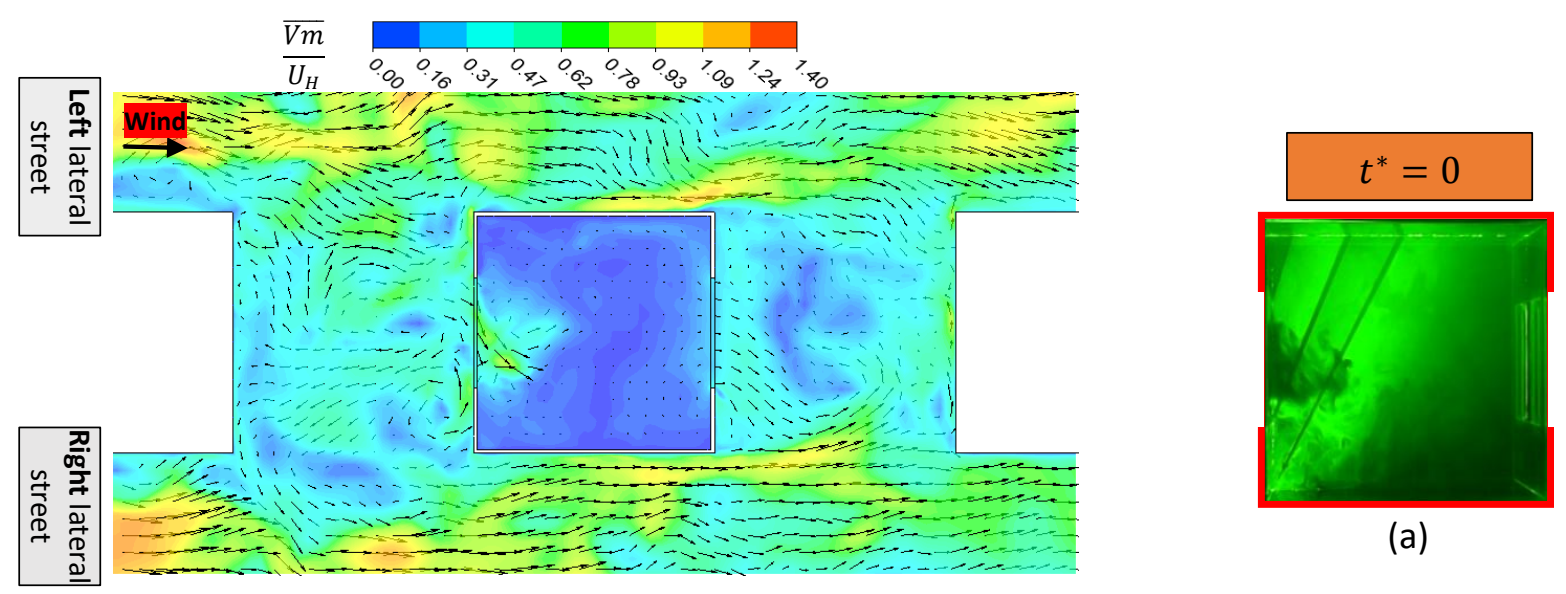

(a)
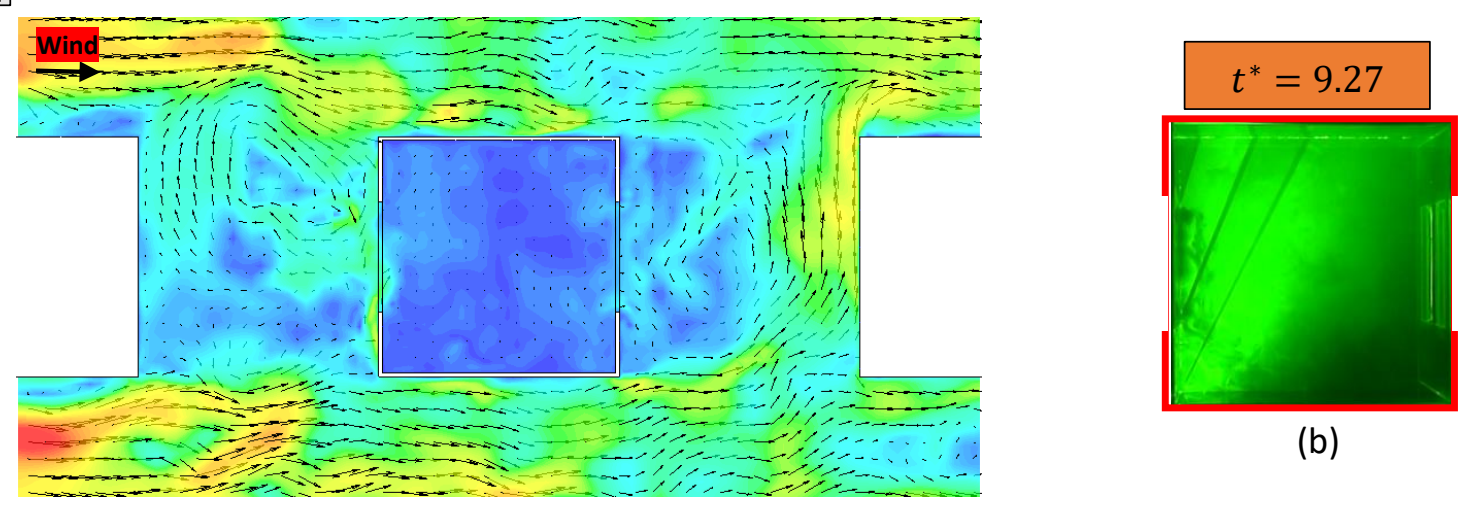

(b)
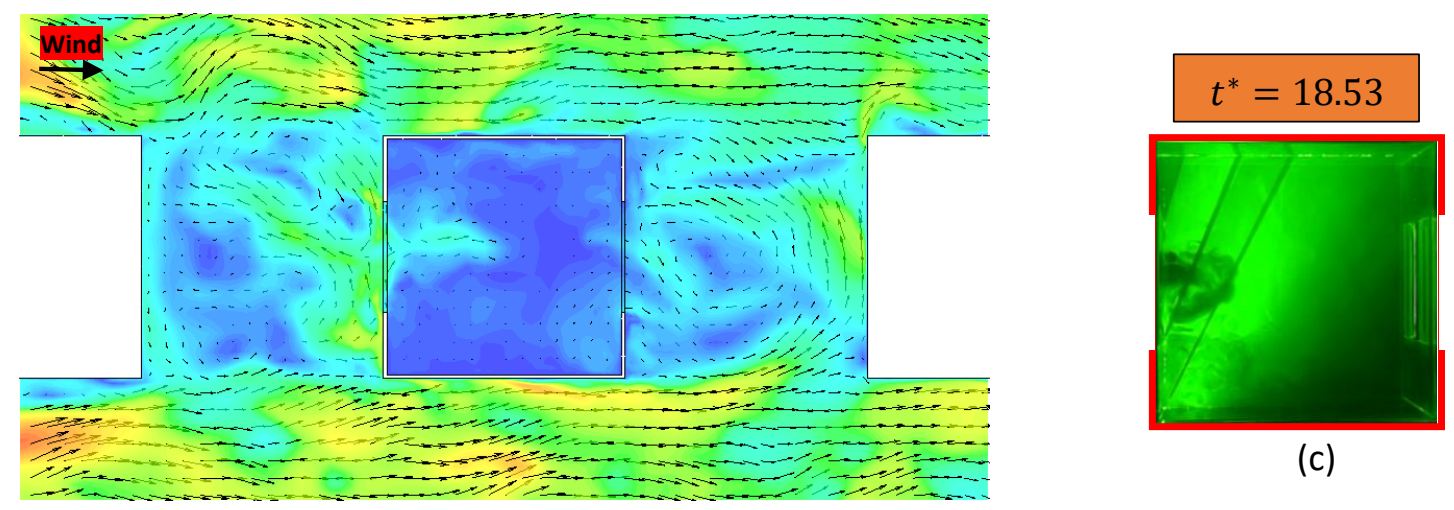

(c)
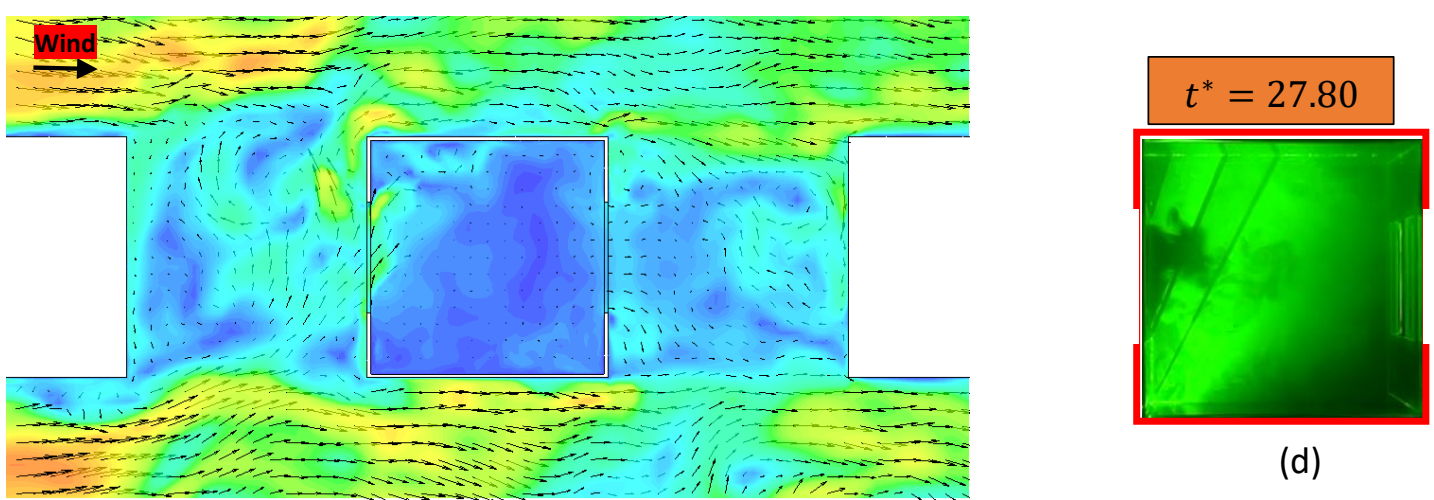

(d)

Figure 14 Instantaneous snapshots of the velocity field over a horizontal plane $\left(\frac{z}{h}=0.5\right)$ at four differentnon-dimensionall times 


\section{Conclusions}

This paper comprehensively compares the results of CFD simulations based on SRANS and LES with those of wind tunnel experiments for a cross-ventilation flow in a group of generic buildings with a regular arrangement. Different turbulence models were considered for the SRANS calculations while the Smagorinsky subgrid-scale model was used for the LES calculations. Comparison of the mean flow structure and turbulence statistics generated by SRANS and LES with the wind tunnel experiments results in the following conclusions:

- $\quad$ SRANS models fail to predict the most important features of cross-ventilation such as windward and leeward jets and flow distribution inside the building regardless of the turbulence models.

- Inability of SRANS in the prediction of the mean flow structure results in a significant deviation in prediction of the crossing airflow rate through the opening and wind surface pressure over the building surfaces.

- Accuracy of LES in the prediction of the mean flow structure, wind surface pressure, and crossing airflow rate through the openings is much higher than the SRANS.

- The high accuracy of LES is attributed to the reproduction of the anisotropic velocity fluctuations of cross-ventilation flow around and inside the target building.

- The anisotropic velocity fluctuations are mainly caused by the transient momentum transfer mechanisms in the street canyon flow, which cannot be reproduced in SRANS.

Application of SRANS for urban airflow modeling and building energy simulations for calculation of cross-ventilation and building surface pressure distribution associated to cross-ventilation flows should be considered with more cautions as it can be significantly unreliable in some instances. Turbulence model calibration and modification in SRANS seem to be necessary in this regard. In this study, a simplified generic building configuration with a constant wind angle was considered for the cross-ventilation analysis based on a wind tunnel measurement, whilst the building arrangement and shape are more complex in the urban areas. Therefore, impacts of the building shape, wind direction, and urban configuration on the cross-ventilation flows should be examined to obtain more general conclusion about the accuracy of SRANS and LES. Hence, future studies should be focused on the investigation of the LES and SRANS accuracy in prediction of the cross-ventilation flow in different urban configurations. Furthermore, proposing practical solutions to increase the accuracy of SRANS based on the model calibration can be investigated in the future studies.

\section{Acknowledgment}

\section{References}

Aflaki, Ardalan, Kamran Hirbodi, Norhayati Mahyuddin, Mahmood Yaghoubi, and Masoud Esfandiari. 2019. "Improving the Air Change Rate in High-Rise Buildings through a Transom Ventilation Panel: A Case Study." Building and Environment 147: 35-49.

Akabayashi, Shin-ichi, Akashi Mochida, Yoshihide Tominaga, Masaahi Yoshida, and Jun Sakaguchi. 1996. "Performance of New Wind Tunnel of Niigata Institute of Technology." Wind Engineers, JAWE 1996 (68): 95-106.

Akamine, Yoshihiko, Takashi Kurabuchi, Masaaki Ohba, Tomoyuki Endo, and Motoyasu Kamata. 2004. "A CFD Analysis of the Air Flow Characteristics at an Inflow Opening." International Journal of Ventilation 2 (4): 431-37. 
Aydin, Yusuf Cihat, and Parham A Mirzaei. 2016. "Wind-Driven Ventilation Improvement with Plan Typology Alteration: A CFD Case Study of Traditional Turkish Architecture." In Building Simulation, 1-16. Springer.

Bazdidi-Tehrani, Farzad, Shahin Masoumi-Verki, Payam Gholamalipour, and Mohsen Kiamansouri. 2019. "Large Eddy Simulation of Pollutant Dispersion in a Naturally CrossVentilated Model Building: Comparison between Sub-Grid Scale Models." Building Simulation 12 (5): 921-41. https://doi.org/10.1007/s12273-019-0525-5.

Celik, I B, Z N Cehreli, and I Yavuz. 2005. "Index of Resolution Quality for Large Eddy Simulations." Journal of Fluids Engineering 127 (5): 949-58.

Chu, Chia-Ren, and Bo-Fan Chiang. 2014. "Wind-Driven Cross Ventilation in Long Buildings." Building and Environment 80: 150-58. https://doi.org/http://dx.doi.org/10.1016/j.buildenv.2014.05.017.

Chu, Chia Ren, Y H Chiu, and Yu-Wen Wang. 2010. "An Experimental Study of Wind-Driven Cross Ventilation in Partitioned Buildings." Energy and Buildings 42 (5): 667-73. https://doi.org/https://doi.org/10.1016/j.enbuild.2009.11.004.

Coceal, O, T G Thomas, I P Castro, and S E Belcher. 2006. "Mean Flow and Turbulence Statistics Over Groups of Urban-like Cubical Obstacles." Boundary-Layer Meteorology 121 (3): 491-519. https://doi.org/10.1007/s10546-006-9076-2.

Etheridge, David. 2015. "A Perspective on Fifty Years of Natural Ventilation Research.” Building and Environment 91: 51-60.

Franke, Jörg. 2007. Best Practice Guideline for the CFD Simulation of Flows in the Urban Environment. Meteorological Inst.

Franke, J., 2010. 2010. "A Review of Verification and Validation in Relation to CWE." In Proceedings 5th International Symposium on Computational Wind Engineering (CWE2010), Chapel Hill, North Carolina, USA.

Franke, Jorg, Antti Hellsten, K Heinke Schlunzen, and Bertrand Carissimo. 2011. "The COST 732 Best Practice Guideline for CFD Simulation of Flows in the Urban Environment: A Summary." International Journal of Environment and Pollution 44 (1-4): 419-27.

Gough, H, T Sato, C Halios, C S B Grimmond, Z Luo, J F Barlow, A Robertson, R Hoxey, and A Quinn. 2018. "Effects of Variability of Local Winds on Cross Ventilation for a Simplified Building within a Full-Scale Asymmetric Array: Overview of the Silsoe Field Campaign." Journal of Wind Engineering and Industrial Aerodynamics 175: 408-18. https://doi.org/https://doi.org/10.1016/j.jweia.2018.02.010.

Gough, Hannah L, Zhiwen Luo, Christos H Halios, M-F King, Catherine J Noakes, C Sue B Grimmond, Janet F Barlow, Roger Hoxey, and Andrew D Quinn. 2018. "Field Measurement of Natural Ventilation Rate in an Idealised Full-Scale Building Located in a Staggered Urban Array: Comparison between Tracer Gas and Pressure-Based Methods." Building and Environment 137: 246-56.

Gousseau, P, Bert Blocken, and G J F Van Heijst. 2013. "Quality Assessment of Large-Eddy Simulation of Wind Flow around a High-Rise Building: Validation and Solution Verification." Computers \& Fluids 79: 120-33.

Guichard, Romain. 2019. “Assessment of an Improved Random Flow Generation Method to 
Predict Unsteady Wind Pressures on an Isolated Building Using Large-Eddy Simulation." Journal of Wind Engineering and Industrial Aerodynamics 189: 304-13.

Heracleous, C, and A Michael. 2019. "Experimental Assessment of the Impact of Natural Ventilation on Indoor Air Quality and Thermal Comfort Conditions of Educational Buildings in the Eastern Mediterranean Region during the Heating Period." Journal of Building Engineering 26: 100917.

Hooff, T van, B Blocken, and Y Tominaga. 2017. "On the Accuracy of CFD Simulations of Cross-Ventilation Flows for a Generic Isolated Building: Comparison of RANS, LES and Experiments." Building and Environment 114 (Supplement C): 148-65. https://doi.org/https://doi.org/10.1016/j.buildenv.2016.12.019.

Hooff, Twan van, Bert Blocken, and Yoshihide Tominaga. 2016. "On the Accuracy of CFD Simulations of Cross-Ventilation Flows for a Generic Isolated Building: Comparison of RANS, LES and Experiments." Building and Environment.

$\mathrm{Hu}$, Cheng-Hu, Takashi Kurabuchi, and Masaaki Ohba. 2005. "Numerical Study of CrossVentilation Using Two-Equation RANS Turbulence Models." International Journal of Ventilation 4 (2): 123-32.

$\mathrm{Hu}$, Cheng-Hu, Masaaki Ohba, and Ryuichiro Yoshie. 2008. "CFD Modelling of Unsteady Cross Ventilation Flows Using LES." Journal of Wind Engineering and Industrial Aerodynamics 96 (10): 1692-1706.

Hua, Cheng-Hu, Masaaki Ohbab, and Ryuichiro Yoshiec. 2006. "CFD Modelling of Cross Ventilation Using Unsteady Methods." Journal of Wind Engineering, no. 108: 255-58.

Ikegaya, N, T Okaze, H Kikumoto, M Imano, H Ono, and Y Tominaga. 2019. "Effect of the Numerical Viscosity on Reproduction of Mean and Turbulent Flow Fields in the Case of a 1: 1: 2 Single Block Model." Journal of Wind Engineering and Industrial Aerodynamics 191: 279-96.

Ikegaya, Naoki, S Hasegawa, and Aya Hagishima. 2019. "Time-Resolved Particle Image Velocimetry for Cross-Ventilation Flow of Generic Block Sheltered by Urban-like Block Arrays." Building and Environment 147: 132-45.

lousef, S, H Montazeri, B Blocken, and P J V van Wesemael. 2017. "On the Use of NonConformal Grids for Economic LES of Wind Flow and Convective Heat Transfer for a WallMounted Cube." Building and Environment 119: 44-61. https://doi.org/https://doi.org/10.1016/j.buildenv.2017.04.004.

James Lo, L, David Banks, and Atila Novoselac. 2013. "Combined Wind Tunnel and CFD Analysis for Indoor Airflow Prediction of Wind-Driven Cross Ventilation." Building and Environment 60: 12-23. https://doi.org/http://dx.doi.org/10.1016/j.buildenv.2012.10.022.

Jiang, Yi, Donald Alexander, Huw Jenkins, Rob Arthur, and Qingyan Chen. 2003. "Natural Ventilation in Buildings: Measurement in a Wind Tunnel and Numerical Simulation with Large-Eddy Simulation." Journal of Wind Engineering and Industrial Aerodynamics 91 (3): 331-53.

Jiang, Yi, and Qingyan Chen. 2003. "Buoyancy-Driven Single-Sided Natural Ventilation in Buildings with Large Openings." International Journal of Heat and Mass Transfer 46 (6): 973-88. https://doi.org/http://doi.org/10.1016/S0017-9310(02)00373-3.

Karava, P, T Stathopoulos, and A K Athienitis. 2004. "Wind Driven Flow through Openings-a 
Review of Discharge Coefficients." International Journal of Ventilation 3 (3): 255-66.

Katayama, T, J Tsutsumi, and A Ishii. 1992. "Full-Scale Measurements and Wind Tunnel Tests on Cross-Ventilation." Journal of Wind Engineering and Industrial Aerodynamics 44 (1-3): 2553-62.

Kato, Shinsuke, Shuzo Murakami, Akashi Mochida, Shin-ichi Akabayashi, and Yoshihide Tominaga. 1992. "Velocity-Pressure Field of Cross Ventilation with Open Windows Analyzed by Wind Tunnel and Numerical Simulation." Journal of Wind Engineering and Industrial Aerodynamics 44 (1-3): 2575-86.

King, Marco-Felipe, Amirul Khan, Nicolas Delbosc, Hannah L Gough, Christos Halios, Janet F Barlow, and Catherine J Noakes. 2017. "Modelling Urban Airflow and Natural Ventilation Using a GPU-Based Lattice-Boltzmann Method." Building and Environment 125: 273-84.

Kobayashi, Tomohiro, Kazunobu Sagara, Toshio Yamanaka, Hisashi Kotani, Shogo Takeda, and Mats Sandberg. 2009. "Stream Tube Based Analysis of Problems in Prediction of Cross-Ventilation Rate." International Journal of Ventilation 7 (4): 321-34.

Kobayashi, Tomohiro, Mats Sandberg, Hisashi Kotani, and Leif Claesson. 2010. "Experimental Investigation and CFD Analysis of Cross-Ventilated Flow through Single Room Detached House Model." J Building Environment 45 (12): 2723-34.

Kosutova, Katarina, Twan van Hooff, Christina Vanderwel, Bert Blocken, and Jan Hensen. 2019. "Cross-Ventilation in a Generic Isolated Building Equipped with Louvers: WindTunnel Experiments and CFD Simulations." Building and Environment 154. https://doi.org/10.1016/j.buildenv.2019.03.019.

Kubota, Tetsu, Masao Miura, Yoshihide Tominaga, and Akashi Mochida. 2008. "Wind Tunnel Tests on the Relationship between Building Density and Pedestrian-Level Wind Velocity: Development of Guidelines for Realizing Acceptable Wind Environment in Residential Neighborhoods." Building and Environment 43 (10): 1699-1708.

Kurabuchi, T, M Ohba, A Arashiguchi, and T Iwabuchi. 2000. "Numerical Study of Airflow Structure of a Cross Ventilated Model Building." Air Distribution in Rooms: Ventilation for Health and Sustainable Environment 2: 313.

Kurabuchi, Takashi, Masaaki Ohba, Tomonobu Goto, Yoshihiko Akamine, Tomoyuki Endo, and Motoyasu Kamata. 2005. "Local Dynamic Similarity Concept as Applied to Evaluation of Discharge Coefficients of Cross-Ventilated Buildings-Part 1 Basic Idea and Underlying Wind Tunnel Tests; Part 2 Applicability of Local Dynamic Similarity Concept; Part 3 Simplified Method for Es." International Journal of Ventilation 4 (3): 285.

Launder, Brian Edward, and D B Spalding. 1974. "The Numerical Computation of Turbulent Flows." Computer Methods in Applied Mechanics and Engineering 3 (2): 269-89.

Li, Zhenzhen, Chao Chen, Le Yan, Song Pan, and Lili Zhang. 2014. “'Cross-Ventilation' Effect of Piston Wind and Energy-Saving Evaluation for the Ventilation and Air Condition in Subway Station BT - Proceedings of the 8th International Symposium on Heating, Ventilation and Air Conditioning." In , edited by Angui Li, Yingxin Zhu, and Yuguo Li, 14756. Berlin, Heidelberg: Springer Berlin Heidelberg.

Lien, Jason, and Noor Ahmed. 2011. "Wind Driven Ventilation for Enhanced Indoor Air Quality." In Chemistry, Emission Control, Radioactive Pollution and Indoor Air Quality. IntechOpen.

Lo, L James. 2011. “CFD Simulation of Cross-Ventilation Using Fluctuating Pressure Boundary 
Conditions." ASHRAE Transactions 117: 621.

Lo, L James, and Atila Novoselac. 2013. "Effect of Indoor Buoyancy Flow on Wind-Driven Cross Ventilation." In Building Simulation, 6:69-79. Springer.

Martins, Nuno R, and Guilherme Carrilho da Graça. 2016. "Validation of Numerical Simulation Tools for Wind-Driven Natural Ventilation Design." In Building Simulation, 9:75-87. Springer.

Menter, Florian R. 1994. "Two-Equation Eddy-Viscosity Turbulence Models for Engineering Applications." AIAA Journal 32 (8): 1598-1605.

Mochida, Akashi, Hiroshi Yoshino, Satoshi Miyauchi, and Teruaki Mitamura. 2006. "Total Analysis of Cooling Effects of Cross-Ventilation Affected by Microclimate around a Building." Solar Energy 80 (4): 371-82.

Mochida, Akashi, Hiroshi Yoshino, Tomoya Takeda, Toshimasa Kakegawa, and Satoshi Miyauchi. 2005. "Methods for Controlling Airflow in and around a Building under CrossVentilation to Improve Indoor Thermal Comfort." Journal of Wind Engineering and Industrial Aerodynamics 93 (6): 437-49. https://doi.org/http://dx.doi.org/10.1016/j.jweia.2005.02.003.

Murakami, S. 1991. "Wind Tunnel Test on Velocity-Pressure Field of Cross-Ventilation with Open Windows." ASHRAE Transactions 97: 525-38.

Murakami, Shuzo, Akashi Mochida, and Yoshihiko Hayashi. 1990. "Examining the $k-\epsilon$ Model by Means of a Wind Tunnel Test and Large-Eddy Simulation of the Turbulence Structure around a Cube." Journal of Wind Engineering and Industrial Aerodynamics 35: 87-100.

Murakami, Shuzo, Ryozo Ooka, Shinsuke Kato, Satoru lizuka, and Akashi Mochida. 1996. "Numerical Prediction of Flow around a Building with Various Turbulence Models: Comparison of \{kappa\}-\{var_epsilon\} EVM, ASM, DSM, and LES Wind Tunnel Tests." American Society of Heating, Refrigerating and Air-Conditioning Engineers ....

Nakajima, Keigo, Ryozo Ooka, and Hideki Kikumoto. 2018. "Evaluation of K- $\varepsilon$ Reynolds Stress Modeling in an Idealized Urban Canyon Using LES." Journal of Wind Engineering and Industrial Aerodynamics 175: 213-28.

Nikas, K-S, N Nikolopoulos, and A Nikolopoulos. 2010. "Numerical Study of a Naturally CrossVentilated Building." Energy and Buildings 42 (4): 422-34.

Ohba, Masaaki, Takashi Kurabuchi, Endo Tomoyuki, Yoshihiko Akamine, Motoyasu Kamata, and Aya Kurahashi. 2004. "Local Dynamic Similarity Model of Cross-Ventilation Part 2Application of Local Dynamic Similarity Model." International Journal of Ventilation 2 (4): 383-94.

Ohba, Masaaki, and Isaac Lun. 2010. "Overview of Natural Cross-Ventilation Studies and the Latest Simulation Design Tools Used in Building Ventilation-Related Research." Advances in Building Energy Research 4 (1): 127-66. https://doi.org/10.3763/aber.2009.0405.

Okaze, T, H Kikumoto, H Ono, M Imano, T Hasama, T Kishida, K Nakao, N Ikegaya, Y Tabata, and Y Tominaga. 2017. "Large-Eddy Simulations of Flow around a High-Rise BuildingValidation and Sensitivity Analysis on Turbulent Statistics." In Proceedings of the 7th European and African Conference on Wind Engineering, Liege, Belgium, 3-6.

Park, J S. 2013. "Long-Term Field Measurement on Effects of Wind Speed and Directional Fluctuation on Wind-Driven Cross Ventilation in a Mock-up Building." Building and 
Environment 62: 1-8. https://doi.org/http://dx.doi.org/10.1016/j.buildenv.2012.12.013.

Perén, J I, T Van Hooff, B C C Leite, and Bert Blocken. 2015. "CFD Analysis of CrossVentilation of a Generic Isolated Building with Asymmetric Opening Positions: Impact of Roof Angle and Opening Location." Building and Environment 85: 263-76.

Perén, J I, Twan van Hooff, B C C Leite, and B Blocken. 2015. "Impact of Eaves on CrossVentilation of a Generic Isolated Leeward Sawtooth Roof Building: Windward Eaves, Leeward Eaves and Eaves Inclination." Building and Environment 92: 578-90.

Pope, Stephen B. 2001. "Turbulent Flows." IOP Publishing.

Ramponi, R, and Bert Blocken. 2012a. "CFD Simulation of Cross-Ventilation Flow for Different Isolated Building Configurations: Validation with Wind Tunnel Measurements and Analysis of Physical and Numerical Diffusion Effects." Journal of Wind Engineering and Industrial Aerodynamics 104: 408-18.

Ramponi, R., Blocken, B. 2012b. "CFD Simulation of Cross-Ventilation for a Generic Isolated Building: Impact of Computational Parameters." Building and Environment 53: 34-48.

Schatzmann, Micheal. 2010. COST 732 Model Evaluation Case Studies: Approach and Results. Meteorological Inst.

Sergent, Emmanuel. 2002. "Vers Une Methodologie de Couplage Entre La Simulation Des Grandes Echelles et Les Modeles Statistiques." Ecully, Ecole centrale de Lyon.

Shih, Tsan-Hsing, William W Liou, Aamir Shabbir, Zhigang Yang, and Jiang Zhu. 1995. "A New K- $\epsilon$ Eddy Viscosity Model for High Reynolds Number Turbulent Flows." Computers \& Fluids 24 (3): 227-38.

Shirasawa, Taichi, Akashi Mochida, Yoshihide Tominaga, and Hiroshi YOSHINO. 2006. "Evaluation of Turbulent Time Scale of Linear Revised k- $\varepsilon$ Models Based on LES Data." JWE: 日本風工学研究会誌, no. 108: 125-28.

Shirzadi, M., P.A. Mirzaei, and M. Naghashzadegan. 2017. "Improvement of K-Epsilon Turbulence Model for CFD Simulation of Atmospheric Boundary Layer around a High-Rise Building Using Stochastic Optimization and Monte Carlo Sampling Technique." Journal of Wind Engineering and Industrial Aerodynamics 171. https://doi.org/10.1016/j.jweia.2017.10.005.

Shirzadi, M., Mirzaei, P.A., Naghashzadegan, M., 2018. "Development of an Adaptive Discharge Coefficient to Improve the Accuracy of Cross-Ventilation Airflow Calculation in Building Energy Simulation Tools." Building and Environment 127. https://doi.org/10.1016/j.buildenv.2017.10.019.

Shirzadi, M., P.A. Mirzaei, M. Naghashzadegan, and Y. Tominaga. 2018. "Modelling Enhancement of Cross-Ventilation in Sheltered Buildings Using Stochastic Optimization." International Journal of Heat and Mass Transfer 118. https://doi.org/10.1016/j.ijheatmasstransfer.2017.10.107.

Shirzadi, M., M. Naghashzadegan, and P. A. Mirzaei. 2018. "Improving the CFD Modelling of Cross-Ventilation in Highly-Packed Urban Areas." Sustainable Cities and Society 37. https://doi.org/10.1016/j.scs.2017.11.020.

Shirzadi, Mohammadreza, Parham A Mirzaei, and Mohammad Naghashzadegan. 2018. 
"Developing a Systematic Framework for Increasing the Accuracy of RANS Models for CFD Simulations of Atmospheric Boundary Layer." The 7th International Symposium on Computational Wind Engineering. South Korea.

Shirzadi, Mohammadreza, Mohammad Naghashzadegan, and Parham A Mirzaei. 2019. "Developing a Framework for Improvement of Building Thermal Performance Modeling under Urban Microclimate Interactions." Sustainable Cities and Society 44: 27-39.

Shirzadi, Mohammadreza, Yoshihide Tominaga, and Parham A. Mirzaei. 2019a. "Wind Tunnel Experiments on Cross-Ventilation Flow of a Generic Sheltered Building in Urban Areas." Building and Environment 158 (July): 60-72.

https://doi.org/10.1016/J.BUILDENV.2019.04.057.

Shirzadi, Mohammadreza, Yoshihide Tominaga, and Parham A Mirzaei. 2019b. "Experimental Study on Cross-Ventilation of a Generic Building in Highly-Dense Urban Areas: Impact of Planar Area Density and Wind Direction." Journal of Wind Engineering and Industrial Aerodynamics, 104030.

Shirzadi, M., Tominaga, Y., Mirzaei, P.A. 2019c. "Wind Tunnel Experiments on CrossVentilation Flow of a Generic Sheltered Building in Urban Areas." Building and Environment 158: 60-72. https://doi.org/https://doi.org/10.1016/j.buildenv.2019.04.057.

Shirzadi, M., Tominaga, Y., Mirzaei, P.A. 2020. "Experimental and Steady-RANS CFD Modelling of Cross-Ventilation in Moderately-Dense Urban Areas." Sustainable Cities and Society 52: 101849.

Straw, M P, C J Baker, and A P Robertson. 2000. "Experimental Measurements and Computations of the Wind-Induced Ventilation of a Cubic Structure." Journal of Wind Engineering and Industrial Aerodynamics 88 (2-3): 213-30.

Tominaga, Yoshihide. 2015. "Flow around a High-Rise Building Using Steady and Unsteady RANS CFD: Effect of Large-Scale Fluctuations on the Velocity Statistics." Journal of Wind Engineering and Industrial Aerodynamics 142: 93-103.

Tominaga, Yoshihide, Shin-ichi Akabayashi, Takuya Kitahara, and Yuki Arinami. 2015. “Air Flow around Isolated Gable-Roof Buildings with Different Roof Pitches: Wind Tunnel Experiments and CFD Simulations." Building and Environment 84: 204-13.

Tominaga, Yoshihide, and Bert Blocken. 2015. "Wind Tunnel Experiments on Cross-Ventilation Flow of a Generic Building with Contaminant Dispersion in Unsheltered and Sheltered Conditions." Building and Environment 92: 452-61.

Tominaga, Yoshihide, and Bert Blocken. 2016. "Wind Tunnel Analysis of Flow and Dispersion in Cross-Ventilated Isolated Buildings: Impact of Opening Positions." Journal of Wind Engineering and Industrial Aerodynamics 155: 74-88.

Tominaga, Yoshihide, Akashi Mochida, Shuzo Murakami, and Satoshi Sawaki. 2008. "Comparison of Various Revised $\mathrm{k}-\varepsilon$ Models and LES Applied to Flow around a High-Rise Building Model with 1: 1: 2 Shape Placed within the Surface Boundary Layer." Journal of Wind Engineering and Industrial Aerodynamics 96 (4): 389-411.

Tominaga, Yoshihide, Akashi Mochida, Ryuichiro Yoshie, Hiroto Kataoka, Tsuyoshi Nozu, Masaru Yoshikawa, and Taichi Shirasawa. 2008. "AIJ Guidelines for Practical Applications of CFD to Pedestrian Wind Environment around Buildings." Journal of Wind Engineering and Industrial Aerodynamics 96 (10): 1749-61. 
Tominaga, Yoshihide, and Ted Stathopoulos. 2011. "CFD Modeling of Pollution Dispersion in a Street Canyon: Comparison between LES and RANS." Journal of Wind Engineering and Industrial Aerodynamics 99 (4): 340-48.

Tsuchiya, M, S Murakami, A Mochida, K Kondo, and Y Ishida. 1997. "Development of a New K$\varepsilon$ Model for Flow and Pressure Fields around Bluff Body." Journal of Wind Engineering and Industrial Aerodynamics 67: 169-82.

Yakhot, Victor, and Steven A Orszag. 1986. "Renormalization-Group Analysis of Turbulence." Physical Review Letters 57 (14): 1722.

Yoshie, R, A Mochida, Y Tominaga, H Kataoka, K Harimoto, T Nozu, and T Shirasawa. 2007. "Cooperative Project for CFD Prediction of Pedestrian Wind Environment in the Architectural Institute of Japan." Journal of Wind Engineering and Industrial Aerodynamics 95 (9): 1551-78.

Zhang, Ruijun, Parham A Mirzaei, and Benjamin Jones. 2018. "Development of a Dynamic External CFD and BES Coupling Framework for Application of Urban Neighbourhoods Energy Modelling." Building and Environment 146: 37-49. 